\title{
Design Analysis of Adaptive Beamforming in a MIMO-millimeter Wave 5G Heterogeneous Wireless Network using Machine Learning Models
}

\section{Sanjeev Chopra ( $\sim$ chopra_sanjeev2006@yahoo.com )}

Thapar Institute of Engineering and Technology https://orcid.org/0000-0002-6915-6746

\section{Research Article}

Keywords: Adaptive Beamforming, Multiple-Input-Multiple-Output, Millimeter- Wave, 5G Machine Learning, Random Forest, Maximum Signal-to-Noise- Interference Ratio

Posted Date: April 13th, 2021

DOI: https://doi.org/10.21203/rs.3.rs-312958/v1

License: (9) This work is licensed under a Creative Commons Attribution 4.0 International License.

Read Full License 
Design Analysis of Adaptive Beamforming in a MIMO-millimeter Wave 5G Heterogeneous Wireless Network using Machine Learning Models

$$
\begin{gathered}
\text { Sanjeev Chopra ECED, T.I.E.T., } \\
\text { Punjab. } \\
\text { schopra_phd17@thapar.edu }
\end{gathered}
$$

\section{Abstract}

Beamforming (BF) is a smart antenna technique to provide a summation of the weighted signal over multi-users to produce the more concentrated transmitted signal from massive MIMO antenna arrays deployed in a Millimeter-Wave (mmWave) 5G heterogeneous wireless network. It adjusts the amplitudes and phases of the signals received over different antennas in an optimum manner in the form of directional radiation. This paper will help in the installation of $5 G$ and $6 \mathrm{G} \mathrm{mm-}$ Wave heterogeneous wireless networks. Here, adaptive BF is designed and being implemented on the Machine Learning (ML) platform using Signal-to-NoiseInterference Ratio (SINR). The four ML methods having six BF properties to estimate the SINR of Multiple-Input-Multiple-Output (MIMO) - mm-Wave 5G wireless network are explored. The proposed algorithm suppresses noise plus interference and can reduce the power consumption. The python package pyArgus focusing on the $\mathrm{BF}$ and direction finding algorithms has been used for 20,000 simulations. The BF features namely noise variance, number of antenna elements, distance between antenna elements, azimuth angular range of receiving array, elevation angular range of receiving array and Direction of Arrival (DOA) of signal i.e. incident angle of Signal-of-Interest (SOI) are used in predicting the SINR. The 10-fold cross-validation experiment is performed to assess the robustness of the best 
predictive method. By conducting the rigorous simulations, it has been observed that Random Forest (RF) method outperforms over the three other ML methods such as Tree model i.e. rpart, Generalized Linear Model (glm) and Neural Network (nnet), which does the prediction inexpensive and faster. The performance analysis parameters' result represents that the prediction of Mean Absolute Error (MAE) by RF is lowest 70.73 in value, and its Accuracy is maximum $86.40 \%$, in value having the acceptable error on the training-testing data set.

Keywords: Adaptive Beamforming, Multiple-Input-Multiple-Output, MillimeterWave, 5G Machine Learning, Random Forest, Maximum Signal-to-NoiseInterference Ratio.

\section{INTRODUCTION}

BF always guides to determine the quality of received signal by an antenna array using SINR in cellular base stations. It is rigorously used to distinguish real-time and nearly real-time data from other predicted data. It improves the link budget of mm-Wave and is used in sensor arrays of various fields such as radar, sonar, medical imaging, 5G vehicular communication systems and audio systems [2-4]. The mm-Wave communication needs a larger number of antennas at the transceiver and a post-filtering technique to minimize the significant propagation path loss specifically by atmospheric absorption and to provide higher power gain in the form of BF. Moreover, hundreds or thousands of antennas can be accommodated at the transceiver due to the small carrier wavelengths at mm-Wave frequencies for a given size of antenna array, which provide the better flexibility of BF, but increase its complexity [5]. BF can be considered as a spatial linear filtering technique in 5G heterogeneous networks [6]. It gives MIMO diversity gain provided by coherent combining of multiple signal paths and in it, the 
radiation pattern of the antenna array is built in the direction of desired user while minimizing the interference for nearby users. The inter-cell interference are suppressed using linear processing schemes in a coordinated BF fashion. The result of appropriate $\mathrm{BF}$ is that the links become isolated in direction, and intercellular interference plays a negligible role than in current small cellular networks. This fact implies that capacity gain in these systems is achieved by point-to-point technologies. Fixed BF is applied to the sources having fixed Angles- of-Arrival (AoAs) and helps in the network planning as well as antenna deployment schemes [7]. In adaptive BF, the weights of the array used are adapted to the changing signal environment in a continuous manner. The fixed or adaptive BF pattern plays an important role in achieving the spatial selectivity [8]. The reliable Channel State Information (CSI) analysis is necessary in mm-Wave massive MIMO systems for near-optimal BF performance. However, acquiring this analysis becomes very cumbersome practically due to much variation in the used channel and the significant numbers of transceiver antenna elements. Since, in a currently smart antenna array structure having an interface as Digital Signal Processing (DSP), BF technique needs a fairly accurate estimate of DOA. High frequency (HF) communication signals received by the array are passed on to Receiver (RX) front end and then to Analog to Digital Converter (ADC) system. The DOA estimation algorithm is applied to analog to digital converted signal samples. The antenna array calculates and optimizes the BF weights so that the output beam will adapt itself to the DOA of SOI. Fig. 1 depicts the general block diagram of the smart antenna array system. 


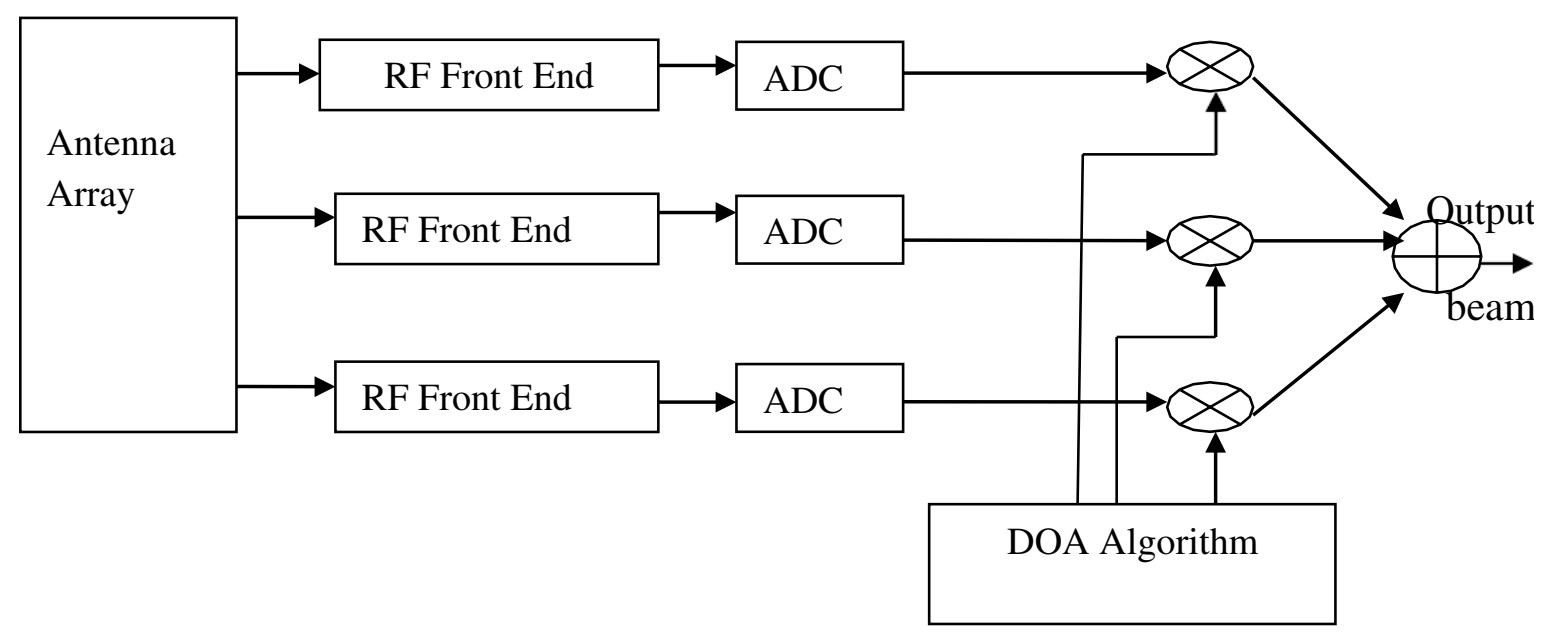

Fig. 1 - General Smart Antenna Array System

The MIMO capability includes several techniques, falling into the categories of BF, diversity, and spatial multiplexing. BF and diversity techniques can reduce the effects of multipath fading, which benefits other communications metrics. Spatial multiplexing can allow multiple independent, parallel data streams to be transmitted, increasing the overall throughput of a system. The availability of large bandwidth in $\mathrm{mm}$-Wave range provides very high frequencies for $5 \mathrm{G}$ mobile communication networks as a promising candidate enabler. To tackle the signal propagation challenge through various paths, mm-Wave systems employ large antenna arrays that are expected to implement highly directional BF and provide higher link-level gains. BF with an antenna array of typically 64 to 512 elements per system within small form factors will reduce interference to adjacent users using a Multi-user (MU)-MIMO system and provides more directivity. In addition to more capacity in the MU-MIMO system, BF has other advantages like reduced energy consumption and the abundant mm-wave spectrum utilization. Its lower energy consumption brings a reduction in overall network operating costs by targeting individual user equipment's with their assigned signals. Full digital baseband precoding is not 
preferred as it has extremely high hardware cost, space and energy utilization in a MIMO system, for the sake of the same number of Radio Frequency (RF) chains. The hybrid analog - digital precoding is a low-cost alternative solution to minimize the number of $\mathrm{RF}$ chains as it divides the precoding operations between the analog and digital domains $[5,9]$. The digital weights of each RF chain are controlled in digital BF. The phase of the signal transmitted at each antenna is adjusted using analog phase shifters in analog BF. Therefore, the hardware-constrained mm-Wave massive MIMO communication system exploits both multiplexing gain and spatial diversity [5].

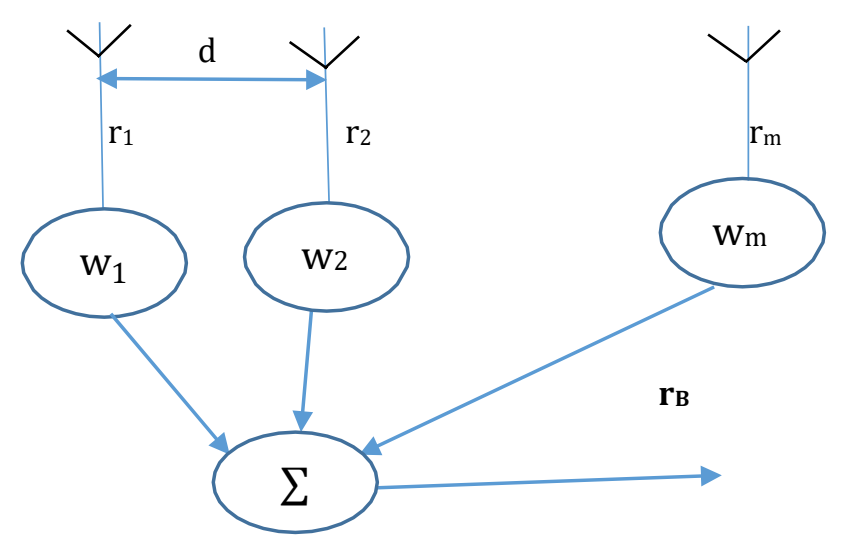

Fig. 2 - Working Principle of Beamformer

The BF adjusts the weights of the antenna elements of the array, which were employed adaptively to optimize the quality of signals under certain performance metrics [10]. From the fig. 2, the BF signal output is calculated using the following equation (1.1):

$$
\mathbf{r}_{B}(t)=w \mathbf{H} \mathbf{r}(\mathbf{t})
$$


where $\mathrm{w}=\left[\begin{array}{lll}\mathrm{w}_{1} & \ldots & \mathrm{w}_{\mathrm{M}}\end{array}\right]^{\mathrm{T}}$ corresponds to the vector of weights of the beamformer proportional to SINR, $\mathrm{r}(\mathrm{t})$ is the array output vector and $\mathrm{H}$ is the channel matrix for a MIMO system described in equation (1.1). $\mathrm{H}$ defines the complex channel gains between the antenna elements of the transmitter as well as of the receiver. It has dimensions $\mathrm{NtXN}_{\mathrm{r}}$, where $\mathrm{N}_{t}$ is the number of transmit antennas, and $\mathrm{N}_{\mathrm{r}}$ is the number of receive antennas. Each value of $\mathrm{H}$ represents the magnitude as well as phase of the channel gain between one pair of transmitter-receiver antenna elements. The matrix is reduced to a one- dimensional vector $\mathrm{h}$, where either a single antenna is assumed on one side of the system, or when the contributions from multiple antennas were combined, such as in the case of receiver diversity techniques, where only the totals at each receiver element are considered [11]. The Quality of Service (QoS) for the receive SNR is given by equation (1.2) which is as follows:

\section{QoS: (Normalized) Receive SNR $=\left|\mathbf{w}^{\mathrm{T}} \mathbf{H}\right|^{2}$}

where temporal variations of $\mathrm{H} \in \mathrm{C}^{\mathrm{N}}$ are the realization of an underlying distribution, but in stochastic approximation case, the analysis of channel distribution is not required; rather most recent channel realization is used. This approximation is well suited for Frequency-Division Duplexing (FDD) systems.

For each receiver $\mathrm{k}$, SINR is calculated using the equation (1.3) which is as follows:

$$
\operatorname{SINR}=\frac{\left|h_{j k}\right|^{2} p_{k}}{\Sigma_{j=k}\left|h_{j k}\right|^{2} p_{k}+\sigma^{2}}
$$

where $h_{j k}$ are the elements of the channel matrix $H, p_{k}$ is the power allocated to the $k$-th link, $\sigma^{2}$ is the nqise power at the k-th receiver. The large value of SINR is essential in the cases of multi-user and multi- relay networks [12]. 


\section{Related Work}

The Multiple Signal Classification (MUSIC) technique, Estimation of Signal Parameters via Rotational Invariance Techniques (ESPRIT), and the Matrix Pencil method and its derivatives, were explained as a part of the BF technique to estimate the DOA of incoming signals based on the peaks of the spatial spectrum by Yang et al., 2010; Liao and Chan, 2011; Oumar et al., 2012; Chuang et al., 2015. However, the computational complexity of MUSIC and ESPRIT methods prevented them to be used in massive arrays. Several studies were done to classify BF techniques according to their physical characteristics especially. Gotsis and Sahalos in 2011 categorized BF techniques mainly as: switched BF and adaptive BF. Moreover, they classified these techniques into circular arrays, linear arrays, and rectangular arrays of array antennas. Hyper BF was categorized as either conventional (switched and fixed) BF or adaptive BF. Switched BF system [13, 14] enhanced SINR of the received signals by choosing one pattern from a lot of predefined patterns. The Butler matrix was developed by Butler and Ralph in 1961 as the most common solution for fixed BF. It depended on a switching network, which chose a suitable beam to obtain the desired signal from a specific terminal.

Various researchers such as Bae et al. in 2014, Huang and Pan in 2015, and Tiwari and Rao in 2015 discussed about the coverage area, interference suppression, capacity and complexity issues of switched BF and adaptive BF. Moreover, a beam typically served more than one Mobile Station (MS) [15]. Fixed BF used a fixed set of weights and time delays to combine the signals using the information mainly about the locations of the sensors in space and the wave direction of interest, received from the sensors employed in the array pattern [16]. Adaptive BF or phased array was based upon the maximization signal at of the desired the main lobe provided by the maximum output SINR of 
beamformer and minimization of the interference signal [17-19]. It was observed that it was a flexible approach to find and estimate the SOI at the output of sensor array using data adaptive spatial or spatio-temporal filtering and interference cancellation. Its performance degradation could also take place, even if the SOI steering vector was precisely known, but the sample size during the training stage was small. Another reason for performance degradation was the environmental non-stationarities because of the fast variations of the propagation channel and rapid motion of interfering sources or antenna arrays. Fangxiao Jin et al. [20] proposed Maximum Correntropy Criterion (MCC) based vigorous cyclic array adaptive BF method to estimate DOA for cyclostationary signals to tackle against the Cycle Frequency Error (CFE) in impulsive noise as well as Gaussian noise environments. Practically, the impulsive noise often shows non-Gaussian properties. It was characterized by sudden bursts and frequently present in wireless systems [21,22]. Due to this, a number of adaptive BF methods such as the Fractional Lower Order Moments-based Beamformer algorithms [23, 24], Linearly Constrained Minimum- 'Normalized Variance' based Beamformer algorithms [25, 26], MCC based Beamformer algorithms [27-29], and Correntropy based Beamformer [30, 31], were provided in the presence of impulsive noise. Taras Maksymyuk et al. discussed an iterative algorithm based on three neural networks for BF in a massive MIMO system, which required an intensive programming for its implementation [32]. Another classification of BF technique based on signal processing to increase the system capacity and performance was presented by Hur et al. in 2013 and Bogale and Le in 2014, wherein the researchers categorized the techniques into analog $\mathrm{BF}$, digital $\mathrm{BF}$, and hybrid $\mathrm{BF}$. The analog BF used the inexpensive phase shifters as a benefit for massive MIMO systems 
compared to digital $\mathrm{BF}$, which has the advantage of providing a more accurate and speedy foundation results to obtain desired user signals. Vishnu V. Ratnam et al. discussed the use of Periodic Analog Channel Estimation (PACE) for designing RX beamformer in massive MIMO systems. In this technique, the channel estimates on one sub-carrier were used to perform $\mathrm{BF}$ on other sub-carriers and was found suitable for sparse channels with $\leq 10$ multipath components in high SNR regime only. The suggested form did not support reception of multiple spatial data streams with more system mismatches. This technique had less loss in $\mathrm{BF}$ gain and a much lower $\mathrm{CE}$ overhead [33]. Kuo-Chen Ho et al. provided two weighted BF algorithms for low and high SNR regimes, which minimized the Symbol Error Rate (SER) for a given transmit power and the transmit power for a given SER. This technique of analog BF supported multiple spatial data transmission with few RF chains on the TX side in an mm-Wave channel model. This technique outperformed the conventional fully-digital zero-forcing BF scheme in terms of hardware cost and quantization effect noise [34]. However, digital BF suffers from high complexity and has an expensive design in massive MIMO systems. Hybrid BF was developed to obtain the advantages of analog as well as digital BF for massive MIMO systems and it employs small number of RF chains. Hybrid BF was classified as partially connected and fully connected. In fully connected type, additional components were used to combine RF signals and provide signal attenuation and power losses. On the other hand, partially connected type used the lesser RF chain access than number of antennas and led to serious drawbacks such as less directivity, wide beamwidth and strong interference from other chains. To have a smaller beam-width, interleaved partially connected array is used currently [35]. Mohammed A. Almagboul 
et al. proposed a partially connected hybrid BF inexpensive receiver based on ImprovedBat (I-BA) algorithm and robust adaptive beamformer in digital domain. I-BA was proposed to avoid the mismatch of DOA by adaptive beamformer and hence, to optimize its weights using the analog phase alignment by linear searching [36]. Hedi Khammari et al. provided an algorithm for allocating the resources as well as for designing the hybrid $\mathrm{BF}$, and discussed K-mean unsupervised ML algorithm for optimal users grouping to reduce the feedback overhead. The proposed ML based analog BF along with zeroforcing digital precoding and user scheduling was used for better performance in terms of sum- rate [37]. Ahmet M. Elbir discussed the hybrid beamformer design in the mm-Wave -MIMO system. It used two Convolutional Neural Networks (CNNs) using the input of channel matrix based upon an optimization problem for the joint design of precoder and combiner. This technique utilized an algorithm for generating the training data for both networks [38]. Lorenzo Combi et al. designed an efficient algorithm for hybrid BF based on the matching-pursuit for a limited-size dictionary of analog beamformers, which was built on the statistical characteristics of the users' distribution. This dictionary was based upon the knowledge of spatial correlation of few DOAs and DODs representing the few radio front-haul channels at mm-Wave frequencies. Since the narrowband processing at the Resource Allocation Unit (RAU) was affected by the beam squint errors, the broadband analog processing with optical delay lines was adopted for HBF [39]. Susnata Mondal et al. demonstrated multi-band MIMO hybrid BF containing multi-antenna Carrier-Aggregation (CA), and RF (or hybrid) beamformer adaptation. This MMSE beam adaptation technique enabled the both main lobe and null adaption [40]. Linlin Sun et al. proposed a robust hybrid analog-and-digital BF scheme, which contained 
Null Space Projection (NSP) in the analog BF domain and Digital Loading (DL) in digital BF domain to oppose DOA estimation errors at RX side [41]. Li Zhu et al. proposed the adaptive hybrid BF based on Dictionary Learning (DL) algorithm for an mm-Wave Line-of-Sight (LoS) MIMO communication system [42]. Yanan Liu et al. discussed the hotspot prediction and beam management for the adaptive Virtual Small Cells (VSCs) in 5G networks. The discussed deep learning technique improved both the cost efficiency and operational efficiency i.e. latency reduction of $5 \mathrm{G}$ networks and managed the beam of VSCs by the way of HBF [43]. Song Noh et al. designed a phaseshifted DFT method based multi-resolution HBF alignment sequence for channel sounding in large-scale mm-Wave - MIMO systems. This sequence designed a subset of codebooks used during the training period. This adaptive design system provided the good performance of average data rate by minimizing the training overhead in the considered mm-Wave systems [44]. Adaptive BF is inconvenient to implement, the major part of present studies related to massive MIMO systems tend to choose BF technique to fixed/switched BF due to its reliability for $5 \mathrm{G}$ requirements. A lot of researchers had implemented the various kinds of adaptive BF algorithms. However, virtually no study was available on adaptive BF in impulsive noise environments (i.e. low SINR) and other wide range of noise environments using ML to our best belief. Conventional featurebased approaches mainly depend upon the expert's knowledge, which may perform well on specialized solutions, but poor in generality and encounter high complexity and timeconsuming. To solve these problems, ML classifiers were shown great advantages. Although ML methods have the advantage of solving classification problems efficiently and good performance, the feature engineering still depends on expert experience 
to some extent, resulting in degradation of accuracy rate. Therefore, self-learning ability is very important in case of an unknown environment. Moreover, ML has a potential to solve the complex problems without explicit programming. Both the research community and industry have advocated the various applications of ML in the field of wireless communication for resource management such as beamformer design, due to its successful applications to many practical tasks like image recognition. To optimize beamformer vectors in a scenario of MIMO broadcast channel, the Weighted Minimum Mean Square Error (WMMSE) algorithm was designed, which transformed the weighted sum-rate maximization problem into a higher dimensional space to make the problem more tractable [45-47].

\section{Problem Statement:}

The suitable BF method increases the signal strength so that it may propagate a large distance through combining various scattered beam components into a single beam in a particular direction with the least affection of environmental conditions. The various researchers had worked on various BF methods, but no one tried to implement any of these methods on the ML platform. The novelty of the proposed work is that we are designing an adaptive or phased array BF system, and implementing as well as analyzing it on the ML platform.

\section{Main Contributions of this paper:}

1. The proposed algorithm is implemented on a ML platform and is analyzed by the four ML methods. 
2. It works in Maximum Signal-to-Noise-Interference Ratio (MSINR) sense by the way of optimal adaptive $\mathrm{BF}$ and is capable of reducing power consumption as well as operational cost in a mm-Wave massive MIMO heterogeneous network by deploying suitable BF method.

3. The SINR responsible for the adaptive $\mathrm{BF}$ under a wide range of noise environments is predicted and maximized against target SINR using the various ML models.

The structure of this paper is as follows:

The introduction along with the related work is presented in section 1 . The types, proposed work flowchart, and data set and methodology of BF are described in section 2. The subsections 2.1.1, 2.1.2 and 2.2 explain transmit BF using Maximum Ratio Transmission (MRT), transmit precoding for BF and proposed work flowchart of adaptive $\mathrm{BF}$ respectively. The considered data set and its features having qualitative assessment, and methodology used are presented in subsection 2.3. The subsection 2.3.1 describes the six relevant features required for the generation of target SINR. The ML methods and model evaluation are presented in section 3. The applicable four ML methods for the prediction of target SINR are discussed in subsection 3.1. Model evaluation using various performance analysis parameters is explained in the subsection 3.2. Section 4 explains simulation results and discussion. The subsection 4.1 is based upon the comparison of performance analysis parameters of the used ML methods. The subsection 4.2 discusses the results of validation and cross-validation simulation experiment. In the last, the conclusion is provided in section 5 . 


\section{Types, Proposed Work Flowchart, Data Set and Methodology of}

\section{BF}

\subsubsection{Transmit BF using MRT}

There are two methods for transmitting: the first is an adaptive technique called MRT and the second technique is precoding. The first technique uses the channel matrix between the transmitting and receiving antennas to optimize power at the receiver. The BF weighting matrix between a transmitter and a particular receiver antenna element, k [48], is defined by the equation (2.1) which is given as follows:

$$
w_{k}=\frac{h_{k} T}{\left|h_{k}\right|}
$$

where $h_{k}$ is the $\mathrm{N}_{\mathrm{t} x} 1$ channel vector between the transmitting array and the receiver antenna. The quantity in the denominator is the absolute magnitude of the channel vector, which is the square root of the sum of the square magnitudes of each of the complex elements of the channel vector. This weighting matrix maximizes the beam to the specific receiver antenna, which is entirely based on the channel vector, scaling both magnitude and phase to form an optimal beam. This beam will likely not represent a specific direction in an environment with a great deal of multipath, and it may include multiple lobes taking advantage of the multipath for the specific transmitter-receiver element combination. When the receiver has more than one element, MRT was applied to form an optimal beam to the first element, and receiver diversity techniques were applied to further enhance the gain using the additional receiver elements.

\subsubsection{Transmit Precoding for BF}

This technique uses a precoding table to specify a collection of predefined beams. The model also provides a mechanism for defining a table of precoding weights that can be 
applied to the H-matrix to perform transmit BF or diversity. The precoding weights provided by Alamouti and pseudo-Alamouti codes increased the probability that a receiver employing MIMO techniques will receive a signal with spatial diversity as an advantage. Precoding tables were used to define codebooks or collections of BF weights that can be used to support a BF method. When the precoding table is used to define multiple sets of precoding weights for each of a number of beams for a massive MIMO antenna array, it is attempted to use each set of weights and will select the set that provides the desired SINR. During a simulation, for each receiver, the model will attempt to use each beam and select the beam that provides the best SINR. This simulates a base station that has a fixed set of beams to choose from, which uses reference signals and feedback from each User Equipment (UE) to select the best beam for transmission to that UE.

\subsection{Proposed Work Flowchart of adaptive BF}

The BF technique is mainly used in radar and sonar systems. Its task is to adjust the array weights to maximize SINR in its output as the source moves, while maintaining a constant gain for the SOI. In BF array, noise and interference are minimized in the output and the beam pattern is optimized by the processor incorporated by adjusting the control weights with respect to a prescribed criterion. BF algorithms were based upon certain criteria like minimizing the variance, maximizing the SINR ratio, minimizing the Mean Square Error (MSE) [49-51], and were used to optimize the smart antenna patterns. The training of the network is performed and control weights are predicted from it in real time. To follow the significant users, 2-D arrays put null values in the direction of the interfering users [52]. The highly directional channel-aware BF supports long outdoor links. The required RF chains are usually lesser to antenna elements. The number of 
available RF chains helps in the realization of a number of beams from a node per time instant. The required performance of a wireless system was achieved, if a beamformer was designed and algorithm alternatives were evaluated as the first step. In the next step, the beamformer was integrated into a system-level model and evaluated over a collection of parameter, steering, and channel combinations. The beam steering helps in a significant reduction of the delayed multipath components. Another cumbersome task is system level tradeoffs between performing BF in the RF domain and/or digital baseband domain. In the proposed work flowchart, an adaptive beamformer is designed with the aims of suppression of noise plus interference and reduction of power consumption. The propagation of mm-Wave is dominated by the LOS component. Hence, the knowledge of AoA of the wave is necessary for its reception at the RX. The optimal weight coefficients of array elements corresponding to all DOA's varying from $\left[-120^{\circ} ; 120^{\circ}\right]$ are calculated/predicted in MSINR sense considering autocorrelation matrix of noise plus interferences and autocorrelation matrix of SOI. These autocorrelation matrices of noise and interferences are not correlated in nature. The necessary phase changes can be carried out with the various digital ICs available today in the market. The SOI autocorrelation matrix is generated by creating the array response vector for SOI. This adaptive algorithm is used for optimal BF in massive MIMO mm-Wave systems and is capable of reducing power consumption by deploying the suitable BF method. The proposed work flowchart of adaptive $\mathrm{BF}$ as shown in fig. 3 is as follows: 


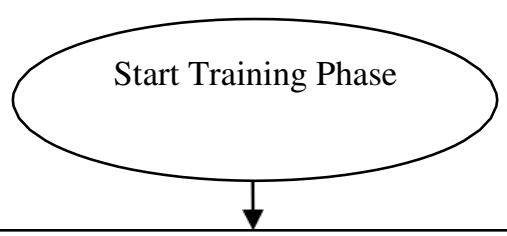

Generate data using pyArgus antenna array pattern, and perform data pre-processing and cleaning in the form of a vector $z^{n}$

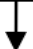

Create the SOI autocorrelation matrix, interference and noise autocorrelation matrices $\left\{\overline{R_{x x}^{n} ;} n=1,2, \ldots . N\right\}$ for all DOA's varying from $\left[-120^{\circ} ; 120^{\circ}\right]$

Predict the control weights of the array elements $\left\{\overline{w_{n}} ; \mathrm{n}=1,2 \ldots, \mathrm{N}\right\}$ corresponding to all DOA's varying from $\left[-120^{\circ} ; 120^{\circ}\right]$ based on following Equation:

$\bar{w}=\mathrm{R}_{\mathrm{xx}}{ }^{-1} \mathrm{~A}\left[\mathrm{~A}^{\mathrm{H}} \mathrm{R}_{\mathrm{xx}}{ }^{-1} \mathrm{~A}\right]^{-1} * \mathrm{f}$; where $A$ points to the desired signals and is called array steering matrix. The term $f$ decides the nature of signals as either interfering or desired signals and updates the weights according to optimum maximum output

Generate input-output pairs $\left\{\left(z^{n} ; \overline{w_{n}}\right)\right.$ for $\left.n=1,2,3 \ldots \mathrm{N}\right\}$, for all sources present in angular range of $\left[-120^{\circ} ; 120^{\circ}\right]$
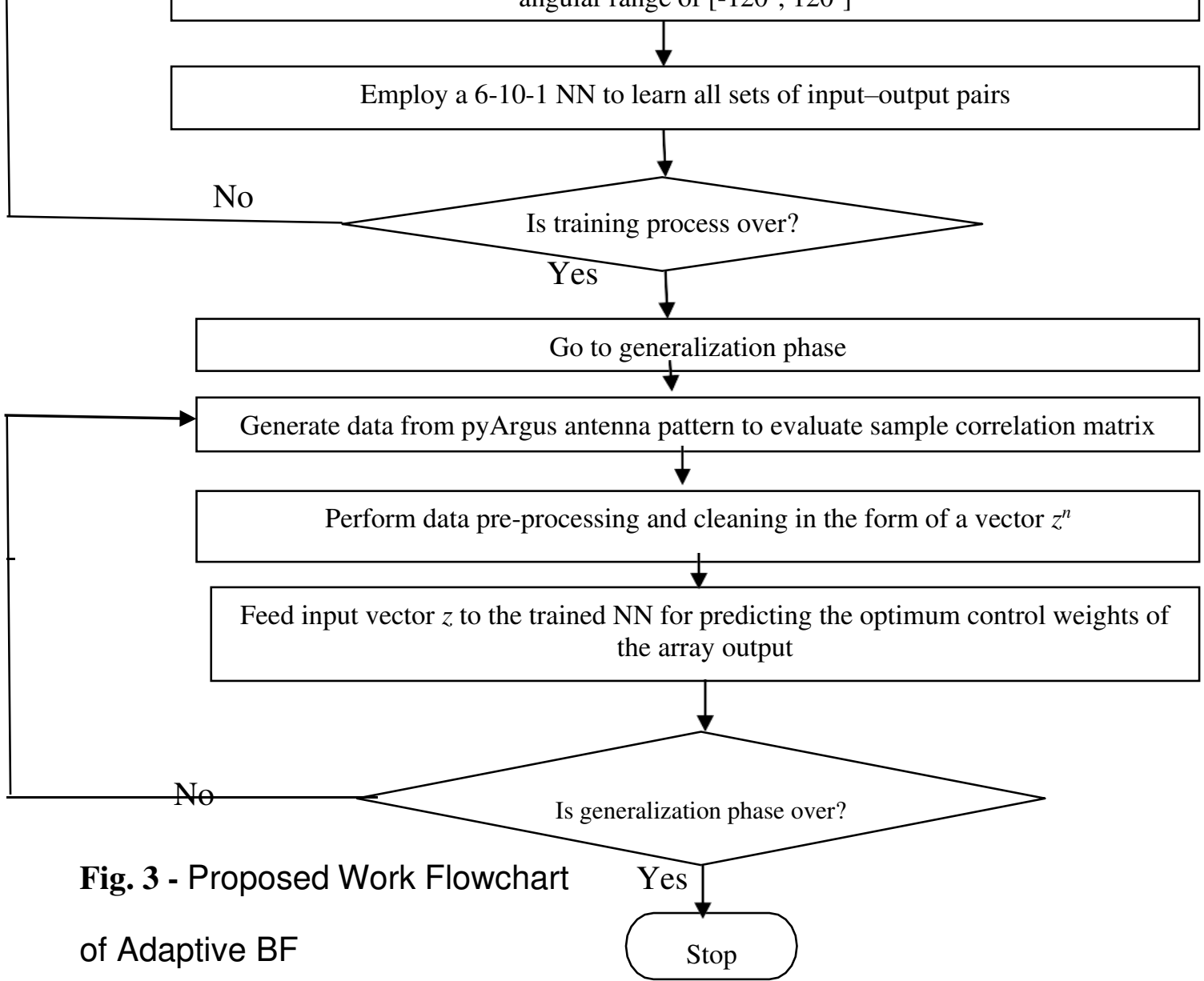


\subsection{Data Set and Methodology}

\subsubsection{Data set and its features having qualitative assessment}

The required data is generated for the work using modeling and simulation. The python package pyArgus [1] has been used for 20,000 simulations. The noise variance, Pnoise is between 0.001 and 0.1 , both are inclusive; $\mathrm{N}$ is the number of elements of an antenna lying between 2 and 10, both are inclusive; $d$ is the distance between the consecutive antenna elements measured in $\lambda$ and lying randomly between 0 and 1(inclusive); $a$ and $b$ are azimuth and elevation angular ranges of receiving array respectively each lying between $-120^{\circ}$ and $+120^{\circ}$, both are inclusive; Theta_soi is SOI lying between- $120^{\circ}$ and $+120^{\circ}$, both are inclusive. The values of a and $\mathrm{b}$ provide a help in the generation of incident angles of interferences. The appropriate value of $\mathrm{d}$ decides the high data rate. More number of radiating elements of the antenna is, more focused of the main lobe of beam is. Table 1 shows a brief description of the features, namely as Pnoise, N, d, a, b and Theta_soi using RF in terms of \% Inc MSE and Inc Node Purity used for the generation of $\mathrm{BF}$ beam in this study. The higher value of any feature plays a more significant role in the generation of BF beams. Table 2 describes the sample dataset used

containing the randomly selected values of all six features. The modeled entries are simulation of python-based signal processing algorithms applicable in antenna arrays (pyArgus). 
Table 1 Description of the features using RF

\begin{tabular}{|c|c|c|}
\hline Feature & Information (\% Inc MSE) & Inc Node Purity \\
\hline$P_{\text {noise }}$ & 148.12 & 1672223101 \\
\hline $\mathrm{N}$ & 72.72 & 315851000 \\
\hline $\mathrm{d}$ & 39.68 & 138440244 \\
\hline $\mathrm{a}$ & 0.74 & 112363333 \\
\hline b & 8.24 & 115403801 \\
\hline Theta_soi & 10.49 & 134960829 \\
\hline
\end{tabular}

\section{Table 2 Sample dataset}

\begin{tabular}{|c|c|c|c|c|c|c|}
\hline $\mathbf{P}_{\text {noise }}$ & $\mathbf{d}$ & $\mathbf{N}$ & theta_soi & $\mathbf{a}$ & $\mathbf{b}$ & SINR \\
\hline 0.053 & 0.3 & 10 & -102 & 117 & -62 & 188.63 \\
\hline 0.018 & 0.2 & 10 & 4 & -8 & -10 & 2.17 \\
\hline 0.028 & 0.9 & 2 & -67 & -89 & -31 & 67.05 \\
\hline 0.016 & 0.5 & 3 & 48 & -96 & -5 & 101.77 \\
\hline 0.094 & 0.1 & 8 & 84 & 117 & 35 & 59.91 \\
\hline 0.071 & 0.4 & 4 & 96 & 21 & 33 & 52.31 \\
\hline 0.09 & 0.9 & 8 & 102 & -14 & 94 & 88.89 \\
\hline 0.033 & 0.8 & 10 & -32 & -81 & 10 & 300.77 \\
\hline 0.023 & 0.7 & 2 & -61 & -10 & -18 & 63.82 \\
\hline 0.058 & 0.4 & 8 & -60 & -73 & -89 & 131.47 \\
\hline 0.091 & 0.8 & 8 & -75 & -70 & 14 & 86.53 \\
\hline 0.061 & 0.6 & 9 & -77 & 10 & -4 & 147.06 \\
\hline 0.027 & 0.2 & 6 & 8 & -87 & -98 & 210.54 \\
\hline 0.007 & 0.6 & 5 & 27 & -9 & 47 & 549.88 \\
\hline 0.042 & 0.7 & 3 & -90 & 17 & 61 & 71.40 \\
\hline 0.051 & 0.7 & 3 & 120 & -46 & -65 & 58.52 \\
\hline 0.095 & 0.4 & 2 & -1 & -111 & -30 & 1.52 \\
\hline 0.042 & 0.5 & 4 & -120 & -107 & 53 & 93.08 \\
\hline 0.07 & 0.7 & 3 & 61 & -81 & 37 & 34.01 \\
\hline
\end{tabular}




\subsubsection{Methodology used}

The methodology of the proposed system, which is used for predicting the target SINR in terms of model evaluation parameters, consists of six steps. These steps as mentioned in Fig. 4 are described as follows:

In step 1, the data is collected through modeling and simulation, and its pre-processing and cleaning is carried out to enhance its accuracy, validity, completeness, consistency and uniformity in step 2 . The step 3 consists of finding the features of importance as mentioned in table 1 of subsection 2.3.1, required to decide their role in the generation of BF beam. In step 4, a model building is done on the pre-processed and cleaned dataset. The step 5 consists of evaluation of the built model using 4 ML methods in terms of performance analysis parameters as mentioned in the table 4 obtained using the equations (3.2) - (3.4) of sub-section 3.2. In step 6, the result is analyzed by the validation and cross-validation simulation experiment as mentioned in sub-section 4.2.

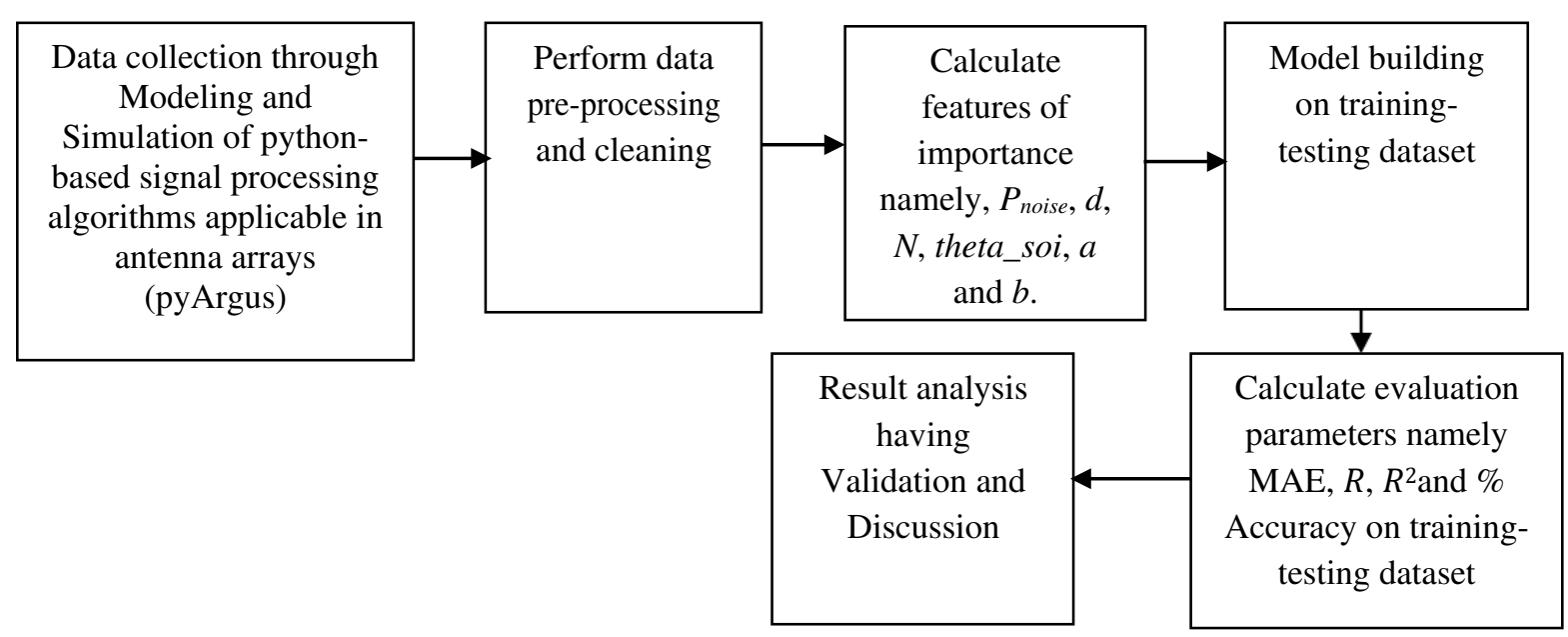

Fig. 4 - Various Steps of Methodology used 


\section{ML Methods and Model Evaluation}

\subsection{Machine Learning (ML) Methods}

The four ML methods (shown in Table 3) used for prediction of SINR of the received signal are present in R open source software, which is licensed under GNU GPL.

\section{Table 3 ML methods used}

\begin{tabular}{|c|c|c|c|}
\hline Model & Method & Package & $\begin{array}{c}\text { Tuning Parameters and } \\
\text { associated Values measured }\end{array}$ \\
\hline $\begin{array}{c}\text { Decision Tree } \\
\text { [53] }\end{array}$ & rpart & rpart & $\begin{array}{r}\text { minSplit }=20, \text { maxDepth }=3, \\
\text { minBucket }=7, \mathrm{n}=14000\end{array}$ \\
\hline $\begin{array}{c}\text { Random Forest } \\
\text { [54] }\end{array}$ & RF & randomForest & $\mathrm{N}_{\text {tree }}=500, \mathrm{~m}_{\text {try }}=2$ \\
\hline LM [55] & Im & glm & none \\
\hline NN [56] & neuralnet & nnet & $\begin{array}{c}h_{\text {layers }}=10, \text { maxNwts. }=87, \\
\text { maxit }=20000\end{array}$ \\
\hline
\end{tabular}

A lot of ML methods are available, but only four methods, namely as rpart, RF, $1 \mathrm{~m}$ and neuralnet are applicable because the proposed model supports only regression and classification data, and output of the proposed model is a numeric value. These methods are explained briefly as:

(a) Decision Tree (rpart): This method is an extension of C4.5 classification algorithms described by Quinlan [53]. It does not support online learning and suffers from easy overfitting problem. Therefore, it is not well suited for the proposed model.

(b) Random Forest (randomForest): It depends upon a dense collection of trees using the inputs of random nature [54]. It supports both types of regression and classification problems having large data sets. It also helps in the identification of most significant 
variables from hundreds of input variables. It is scalable in nature to any number of dimensions and has generally quite acceptable performances. $\mathrm{N}_{\text {tree }}$ and $\mathrm{m}_{\text {try }}$ are the number of trees and number of variables tried at each split, respectively.

(c) Linear Model (glm): It carries out regression, single stratum analysis of variance and analysis of covariance [55].

(d) Neural Network (nnet): Training of neural network is done using back-propagation, resilient back-propagation with or without weight or the modified globally convergent version [56]. The neural network accepts the weights of connections between neurons. When all weights are trained, it is used to predict the class or quantity. hlayers, maxNwts. and maxit are the number of hidden layers, maximum network weights and maximum number of iterations, respectively. The neuralnet used is 6-10-1 network. The tuning parameters used in each method minimize the error.

\subsection{Model Evaluation}

Adaptive beamformers are evaluated in terms of the beamformer response, the output SINR, the array gain, the array sensitivity, and the white noise gain.

The output SINR is determined by the equation (3.1) which is as follows:

$$
\operatorname{SINR}=\left(\mathbf{w}^{\mathrm{H}} \mathbf{R s s} \mathbf{w}\right) /\left(\mathbf{w}^{\mathrm{H}} \mathbf{R}_{(\mathbf{i}+\mathbf{n})} \mathbf{w}\right.
$$

where $\mathrm{R}_{\mathrm{ss}}$ is sample covariance matrix of the source observed at the array beamformer, $\mathrm{R}_{\mathrm{i}+\mathrm{n}}$ is the covariance matrix of the interference and noise considered together. The adaptive beamformer is evaluated by the output SINR only, which provides a measure of the quality of communication, and estimates the ratio between the SOI and noise plus interference. It is optimized under wide range of noise environments by optimizing the value of spacing between elements, element tapering, lattice structure between elements 
and increasing the number of elements in antenna array used. The BF model is evaluated in terms of the following 4 performance analysis parameters given by equations (3.2) (3.4) [57], which are not derived here:

\subsubsection{Mean Absolute Error (MAE)}

It measures the error rate of a regression model. However, it can only be compared between models having errors in the same units [57]. It is calculated by the equation (3.2):

\section{MAE $=$ Average of Absolute value \\ $\left(p_{i}-a_{i}\right)$ for $n$ number of iterations}

where $a_{i}$ is $i^{\text {th }}$ actual SINR target value and $p_{i}$ is $i^{\text {th }}$ predicted SINR target value. It is calculated for all ML methods, which are shown in table 4.

\subsubsection{Correlation $(R)$}

It provides the statistical relationships between true and predicted values. It is defined by the equation (3.3):

$$
\mathbf{R}=\frac{\sum_{i=1}^{n}(x-\bar{x})(y-\bar{y})}{\sqrt{\sum_{i=1}^{n}(x-\bar{x})^{2} \sum_{i=1}^{n}(y-\bar{y})^{2}}}
$$

where $\mathrm{x}$ is the true value, $\mathrm{y}$ is the predicted value, $\bar{x}$ is the average of the all true values, $\bar{y}$ is the average of the all predicted values and $\mathrm{n}$ is the number of iterations. Correlation is present between 0 and 1, and is considered as good if its value approaches 1[57]. It is calculated for all ML methods, which are shown in table 4. 


\subsubsection{Coefficient of Determination $\left(R^{2}\right)$}

It evaluates the proportion of variance of the dependent variable, provided by the regression model and provides its explanatory power [57]. For perfectness of the model $\mathrm{R}^{2}$ is 1 , and for its failure, $\mathrm{R}^{2}$ is zero. It is calculated by taking the square of the $\mathrm{R}-$ value between the predicted and observed values for all ML methods, which are shown in table 4.

\subsubsection{Accuracy}

Training Loss and accuracy give overall measures of the model's performance. The accuracy is improved by preprocessing the data. It is calculated by the following equation (3.4) as percentage deviation of predicted target with true target with some acceptable

error:

$$
\begin{aligned}
\text { Accuracy } & =\frac{100}{n} \sum_{1=1}^{n} q_{i} \\
q_{i} & =\left\{1 \text { if abs }\left(a_{i}-p_{i}\right) \leq e\right. \\
0 & \text { elsewhere, }
\end{aligned}
$$

where $\mathrm{a}$ is the true target, $\mathrm{p}$ is the predicted target, $\mathrm{n}$ is the total number of iterations and e is the acceptable error [57]. It is calculated for all ML methods, which are shown in table 4 .

\subsubsection{K-Fold Cross Validation}

It measures the robustness of the predictive method employed. The generated dataset is randomly divided into say k equal size subsamples as a first step. Thereafter, out of the 
k Sub-samples, a single subsample is retained as the validation data for testing the method, and the remaining $\mathrm{k}-1$ subsamples are used for carrying out the training of the generated data. The cross-validation process is then repeated K-fold of times, with each of the k subsamples used exactly once as the validation data. Then, all the results from Kfolds can be averaged to provide a single estimation. The 10 -fold validation and crossvalidation in terms of true and predicted values of target SINR are shown in figs. 7 and 8.

\section{Simulation Results and Discussion}

The proposed adaptive BF system is hybrid in a sense that it is a combination of an analog part driven by a computer controlled system and a ML part. The prediction results of all employed ML methods on the training-testing data set are analyzed. All the models, which were discussed in section 4 , have been run on a sample dataset (shown in Table 2) and evaluated on correlation, $\mathrm{R}^{2}, \mathrm{MAE}$ and $\%$ accuracy. The dataset is handling a smaller number of input features, which are larger in observation values. The 10 -fold validation is used to assess the robustness of the best predictive method. The regression model suffers from overfitting problem as the criterion used for its training is not exactly the same as the criterion used to judge its efficacy. So, the validation experiment has been conducted on the generated dataset using best predictive model selected from training-testing experiment. The overfitting issue may have less chance, if the number of parameters in the employed network is much smaller as compared to the total number of data points in the training set. If the size of the training dataset is increased by collecting more data, techniques like regularization and early stopping are not feasible to prevent over-fitting. 


\subsection{Training-Testing Simulation Experiment}

The generated data set is divided into two sets - one set is used for training first and thereafter; the second set is used to test the performance of the result. The generated data set is distributed to $70 \%$ and $30 \%$ respectively for all employed methods in trainingtesting experiment. Table 4 depicts the comparative performance of all used methods in the prediction of SINR on correlation, $\mathrm{R}^{2}, \mathrm{MAE}$ and \% accuracy. The performance results as shown in figs. 5 and 6 shows that RF method outperforms over the other three ML methods employed in the prediction of target SINR as there is the closer; more positive and linear relationship between true and predicted values as compared to other three ML models. Fig. 5 (b) and fig. 6 (b) depicts the scatter plot between predicted value and observed value of target SINR on training and testing dataset respectively using the best RF model. The SINR of the received signal can be increased by BF technique. 

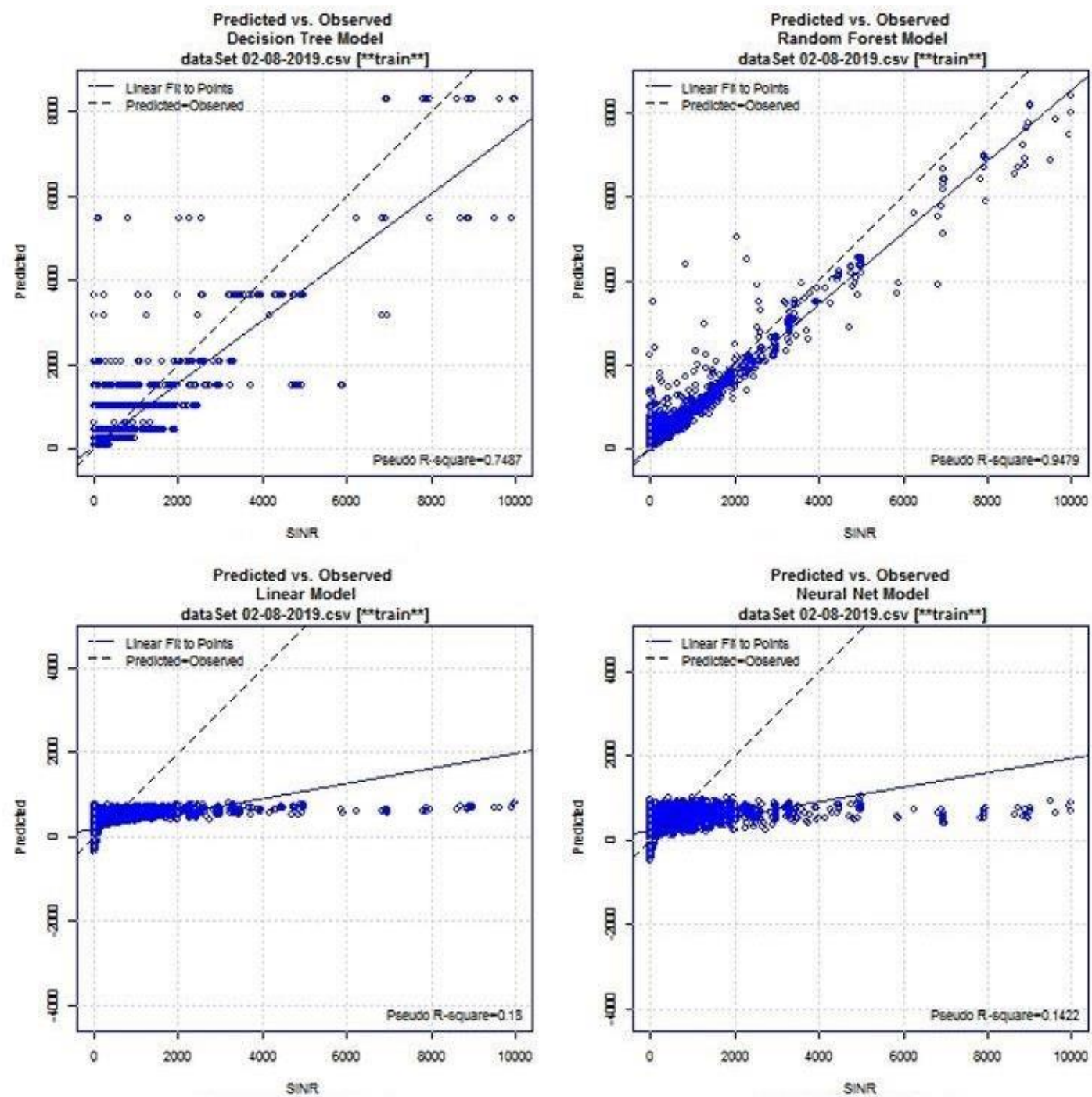

Fig. 5 (a) Predicted vs. Observed Decision Tree Model (b) Predicted vs. Observed Random Forest Model (c) Predicted vs. Observed Linear Model (d) Predicted vs. Observed Neural Net Model on Training dataset 

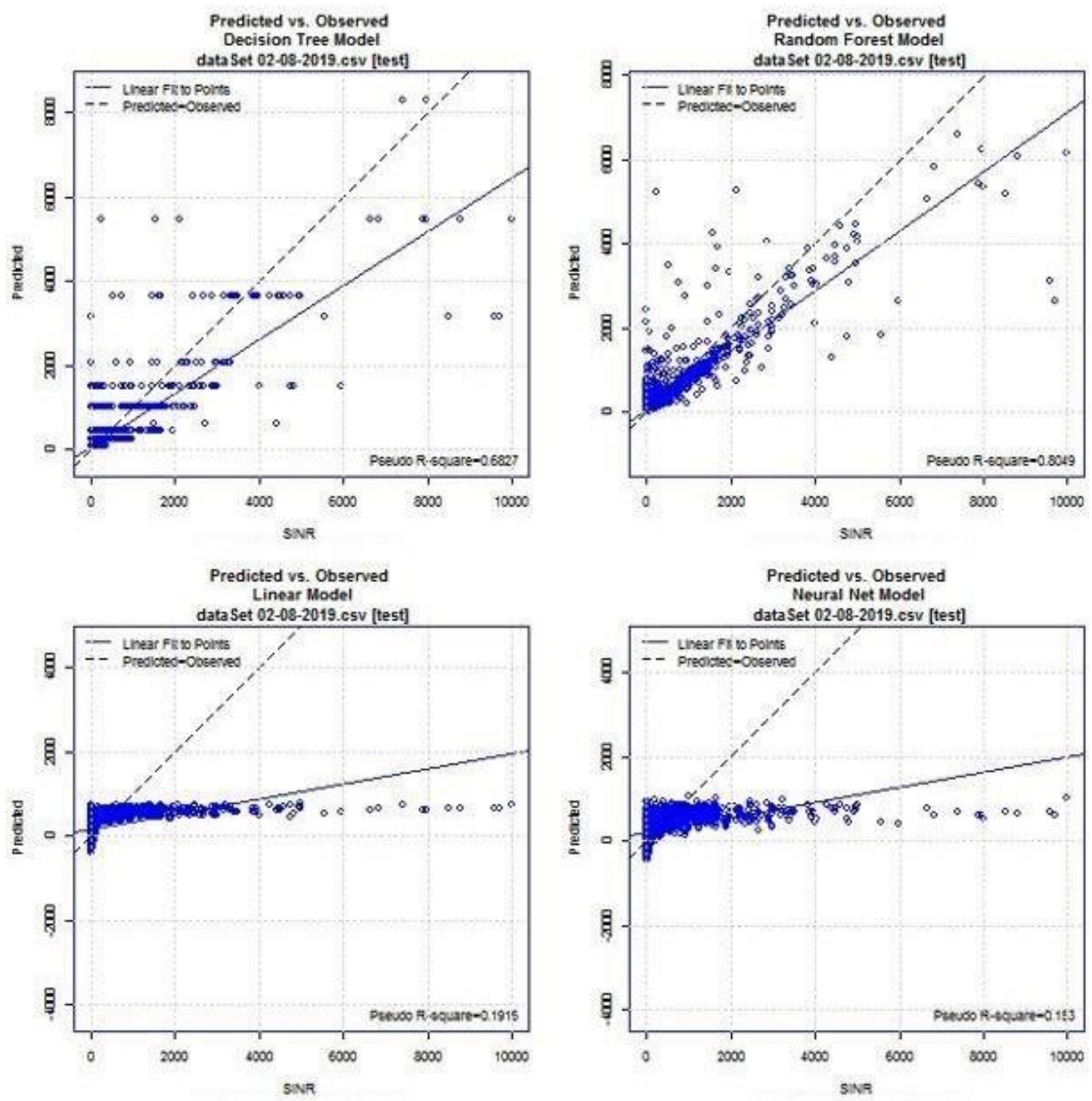

Fig. 6 (a) Predicted vs. Observed Decision Tree Model (b) Predicted vs. Observed Random Forest Model (c) Predicted vs. Observed Linear Model (d) Predicted vs. Observed Neural Net Model on Testing dataset 
Table 4 Performance comparison of employed ML methods as shown in table 3 in the prediction of $R, R^{2}$, MAE and \% Accuracy on training-testing dataset

\begin{tabular}{|c|c|c|c|c|}
\hline \multirow{2}{*}{ Model used } & \multicolumn{4}{|c|}{ Performance Analysis Parameters } \\
\cline { 2 - 5 } & $\mathbf{R}$ & $\mathbf{R}^{\mathbf{2}}$ & MAE & \% Accuracy \\
\hline $\begin{array}{c}\text { Decision } \\
\text { Tree [53] }\end{array}$ & 0.87 & 0.76 & 122.52 & 72.97 \\
\hline $\begin{array}{c}\text { Random } \\
\text { Forest [54] }\end{array}$ & 0.92 & 0.85 & 70.73 & 86.40 \\
\hline LM [55] & 0.42 & 0.18 & 208.18 & 36.12 \\
\hline NN [56] & 0.20 & 0.04 & 211.53 & 42.50 \\
\hline
\end{tabular}

The MAE is used to measure the average of absolute values of difference values between predicted and true values. It is computed using equation (3.2) and Table 4 depicts the MAE of four employed methods. It has been found that the RF model has the lowest MAE of 70.73 as compared to the other three models on the training-testing dataset. The $R$ value is computed using equation (3.3) and Table 4 presents the $R$ value of the employed methods. It has been observed that the RF model has the largest $\mathrm{R}$ value of 0.92. The $\mathrm{R}^{2}$ parameter is computed by taking the square of correlation and Table 4 presents the $\mathrm{R}^{2}$ parameter of the employed methods. It has been found that the RF model has the largest $\mathrm{R}^{2}$ of 0.85 in the prediction of target SINR on the training-testing dataset. Accuracy is computed using equation (3.4) with some acceptable error and Table 4 depicts the \% accuracy of the employed methods. It has been observed that the RF model has the largest accuracy of $86.40 \%$ having acceptable error in the prediction of target SINR on the training-testing dataset. 
Table 5 Comparison of BF results based on ML of various researchers and our paper

\begin{tabular}{|c|c|c|c|c|c|c|c|}
\hline $\begin{array}{c}\text { Paper/ } \\
\text { Author(s) }\end{array}$ & \multirow[b]{2}{*}{$\begin{array}{c}\text { [53]/J. R. } \\
\text { Quinlan } \\
\text { (1986) }\end{array}$} & \multirow{2}{*}{$\begin{array}{c}\text { [57] / } \\
\text { Prashant } \\
\text { Singh Rana } \\
\text { et al. (2014) }\end{array}$} & \multirow[b]{2}{*}{$\begin{array}{l}\text { [38] Ahmet } \\
\text { M. Elbir } \\
\text { (2019) }\end{array}$} & \multirow[b]{2}{*}{$\begin{array}{c}\text { [58] } \\
\text { Francisco } \\
\text { Hugo Costa } \\
\text { Neto et al. } \\
\text { (2019) }\end{array}$} & \multirow[b]{2}{*}{$\begin{array}{c}\text { [59] } \\
\text { Hyung } \\
\text { Jun Kwon } \\
\text { et al. } \\
\text { (2019) }\end{array}$} & \multirow[b]{2}{*}{$\begin{array}{c}\text { Results of } \\
\text { the } \\
\text { proposed } \\
\text { work }\end{array}$} & \multirow[b]{2}{*}{$\begin{array}{l}\text { Salient } \\
\text { remarks }\end{array}$} \\
\hline $\begin{array}{c}\text { Type of } \\
\text { model/Essential } \\
\text { Simulation } \\
\text { Conditions/ } \\
\text { Parameter(s) }\end{array}$ & & & & & & & \\
\hline Type of Model used & $\begin{array}{c}\text { Complex } \\
\text { decision tree }\end{array}$ & $\begin{array}{l}\text { ML based } \\
\text { modeled } \\
\text { protein } \\
\text { structure } \\
\text { which was } \\
\text { based on } \\
\text { RMSED- } \\
\text { prediction } \\
\text { model }\end{array}$ & $\begin{array}{l}\text { CNN } \\
\text { based } \\
\text { frame } \\
\text { work }\end{array}$ & $\begin{array}{c}\text { The downlink } \\
\text { of a massive- } \\
\text { MIMO } \\
\text { system }\end{array}$ & ML model & $\begin{array}{l}\text { Using the } \\
\text { best RF } \\
\text { model, the } \\
\text { proposed } \\
\text { work is } \\
\text { having the } \\
\text { following } \\
\text { performan } \\
\text { ce analysis }\end{array}$ & $\begin{array}{l}\text { 1. The } \\
\text { dataset of } \\
\text { paper [53] is } \\
\text { little bit more } \\
\text { correlated in } \\
\text { nature, but its } \\
\text { accuracy is } \\
\text { much lower } \\
\text { than the }\end{array}$ \\
\hline $\begin{array}{c}\text { Essential Simulation } \\
\text { Conditions/ } \\
\text { Attributes used }\end{array}$ & $\begin{array}{l}\text { Outlook, } \\
\text { temperature, } \\
\text { humidity and } \\
\text { windy at } 100 \%\end{array}$ & $\begin{array}{l}\text { Physicoche } \\
\text { mical } \\
\text { properties }\end{array}$ & $\begin{array}{c}N_{R}=N_{T}=36, \\
N_{S}=3, \\
\text { Uniform } \\
\text { square arrays } \\
\text { with } 0.5 \wedge \\
\text { spacing, } \\
N^{R F}=N_{R}^{R F}= \\
4, \\
\text { No. of } \\
\text { realizations } \\
\text { of }\end{array}$ & $\begin{array}{c}\text { Angle } \\
\text { sector }=60^{\circ}, \mathrm{BS} \\
\text { height }=10 \mathrm{~m} \text {, } \\
\text { UE height }=1.5 \\
\mathrm{~m}, \mathrm{UE} \\
\text { track=linear, } \\
\text { UEs speed =3 } \\
\mathrm{km} / \mathrm{h}, \mathrm{BS} \\
\text { antenna } \\
\text { model }= \\
\text { (3GPP)- }\end{array}$ & $\begin{array}{l}\text { having } 17 \\
\text { input } \\
\text { nodes, } \\
M \text { hidden } \\
\text { layers with } \\
N \text { hidden } \\
\text { nodes and }\end{array}$ & $\begin{array}{c}\text { parameters } \\
\text { : } \\
\text { Correlation } \\
-0.92 \\
R^{2}-0.85\end{array}$ & $\begin{array}{l}\text { accuracy of } \\
\text { the proposed } \\
\text { work. }\end{array}$ \\
\hline
\end{tabular}




\begin{tabular}{|c|c|c|c|c|c|c|c|c|}
\hline & & noise & & $\begin{array}{c}\text { channel } \\
\text { matrices, } N= \\
\text { No. of noisy } \\
\text { channel matrics, } \\
L=100 \text {, all } \\
\text { transmit and } \\
\text { receive azimuth } \\
\text { and elevation } \\
\text { angles which } \\
\text { were uniform } \\
\text { randomly } \\
\text { selected from the } \\
\text { interval }\left[-60^{\circ}\right. \\
60^{\circ} \text { ] and }\left[-20^{\circ},\right. \\
\left.20^{\circ}\right] \text { respectively }\end{array}$ & $\begin{array}{c}\text { mm-Wave, } \\
\text { BS vertical } \\
\text { antennas=8, } \\
\text { BS horizontal } \\
\text { antennas=8, } \\
\text { BS element } \\
\text { array } \\
\text { spacing=0.5 } \\
\text { ᄉ m, UE } \\
\text { antenna } \\
\text { model=omni } \\
\text {, UE } \\
\text { antennas=1, } \\
\text { no. of } \\
\text { simulation } \\
\text { rounds=50 }\end{array}$ & $\begin{array}{l}4 \text { output } \\
\text { nodes }\end{array}$ & $\begin{array}{c}\text { Mean } \\
\text { Absolute } \\
\text { Error- } \\
70.73 \\
\text { and } \\
\% \\
\text { Accuracy- } \\
86.40, \text { as } \\
\text { shown in } \\
\text { table } 4 \text {. }\end{array}$ & $\begin{array}{l}\text { 2. The } \\
\text { accuracy of } \\
\text { the proposed } \\
\text { method } \\
\text { mainly } \\
\text { depends } \\
\text { upon the } \\
\text { type of the } \\
\text { problem } \\
\text { considered, } \\
\text { and a way of } \\
\text { dataset } \\
\text { collected and } \\
\text { its features of } \\
\text { importance. }\end{array}$ \\
\hline \multirow{2}{*}{ 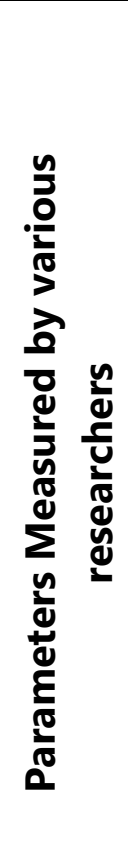 } & 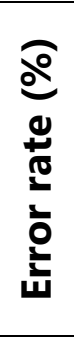 & $\begin{array}{l}\text { Error rate of } \\
\text { all attribute } \\
\quad s=25.9 \%\end{array}$ & $\begin{array}{c}\text { Not } \\
\text { considered }\end{array}$ & $\begin{array}{c}\text { Not } \\
\text { considered }\end{array}$ & $\begin{array}{c}\text { Not } \\
\text { considered }\end{array}$ & $\begin{array}{c}\text { Not } \\
\text { considered }\end{array}$ & & \multirow{2}{*}{$\begin{array}{l}\text { 3. No other } \\
\text { researcher had } \\
\text { considered all } \\
\text { ML methods as } \\
\text { well as no } \\
\text { researcher had } \\
\text { implemented } \\
\text { the designed } \\
\text { BF algorithm } \\
\text { on } \\
\text { platform. }\end{array}$} \\
\hline & 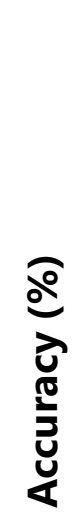 & $\begin{array}{c}\text { Not consider } \\
\text { ed }\end{array}$ & $\begin{array}{c}\text { RMSED } \\
\text { based } \\
78.82 \%\end{array}$ & $\begin{array}{c}\text { Not } \\
\text { considered }\end{array}$ & $\begin{array}{c}\text { Not } \\
\text { considered }\end{array}$ & $\begin{array}{c}\text { Not } \\
\text { considered }\end{array}$ & & \\
\hline
\end{tabular}




\begin{tabular}{|c|c|c|c|c|c|}
\hline 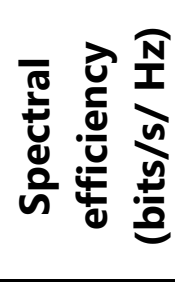 & $\begin{array}{c}\text { Not consider } \\
\text { ed }\end{array}$ & $\begin{array}{c}\text { Not } \\
\text { considered }\end{array}$ & $\begin{array}{c}\text { (5 - 40) of HBF } \\
\text { (Deep } \\
\text { Learning) DL } \\
\text { vs. SNR } \\
(-20-20) d B\end{array}$ & $\begin{array}{c}\text { Not } \\
\text { considered }\end{array}$ & $\begin{array}{c}\text { Not } \\
\text { considered }\end{array}$ \\
\hline$\sum_{z}^{\text {Un }}$ & $\begin{array}{c}\text { Not consider } \\
\text { ed }\end{array}$ & $\begin{array}{c}\text { Not } \\
\text { considered }\end{array}$ & Not considered & $\begin{array}{l}\text { (approx. 10- } \\
2.5 \text { - less than } \\
10-4 \text { ) of the } \\
\text { estimated } \\
\text { channel } \\
\text { vector for } \\
\text { pilot } \\
\text { sequence } \\
\text { length = } 128 \\
\text { vs. SNR (0 - } \\
\text { 20) dB of the } \\
\text { beam }\end{array}$ & $\begin{array}{c}\text { Not } \\
\text { considered }\end{array}$ \\
\hline 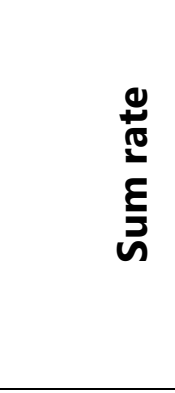 & $\begin{array}{c}\text { Not consider } \\
\text { ed }\end{array}$ & $\begin{array}{c}\text { Not } \\
\text { considered }\end{array}$ & Not considered & $\begin{array}{c}\text { Not } \\
\text { considered }\end{array}$ & $\begin{array}{c}\text { (2 - approx. } \\
15) \text { of the } \\
\text { TXs for } \\
1000000 \\
\text { samples vs. } \\
\text { SNR }(0-25) \\
\text { dB }\end{array}$ \\
\hline
\end{tabular}


Our accuracy result is better than the Root Mean Square Deviation (RMSD) - based result as shown in table 5 having the acceptable error on the training-testing data set of the research paper [57]. The electronically steered BF applied and the corresponding results obtained as shown in tables 4 and 5 play an important role in BS antennas providing the super-high spectrum efficiency through spatial multiplexing, data rate, energy efficiency, network capacity and throughput for near-instant and full unlimited connectivity human-like intelligent $6 \mathrm{G}$ wireless networks. The network throughput can be increased by providing hundreds of beams serving a large number of users at the same time in the form of massive-user MIMO in future $6 \mathrm{G}$ wireless networks.

\subsection{Validation and Cross-Validation Simulation Experiment}

The 10-fold validation and cross-validation are used to measure the robustness of the RF model. Fig. 7 depicts the scatter plot between true and predicted values of target SINR for 10 folds in the validation experiment and this experiment is performed on $15 \%$ of the generated dataset. The pseudo R-square value of 0.8675 from fig. 7 is very close to the pseudo R-square value of 0.8049 from fig. 6 (b). It makes sure that data set used is logical, complete and within acceptable limits. Cross-validation result as shown in fig. 8 depicts the uniform performance on all evaluation parameters of the model. This result is obtained by plotting the scatter plot between actual SINR and predicted SINR of RF model and this plot resembles the validation scatter plot as shown in fig. 7 to a much greater extent. It has been used to better estimate the test error of any model and puts better confidence in the prediction accuracy of the model. It prevents the model over-fitting and gives our model the opportunity to train on a number of train-test splits. 


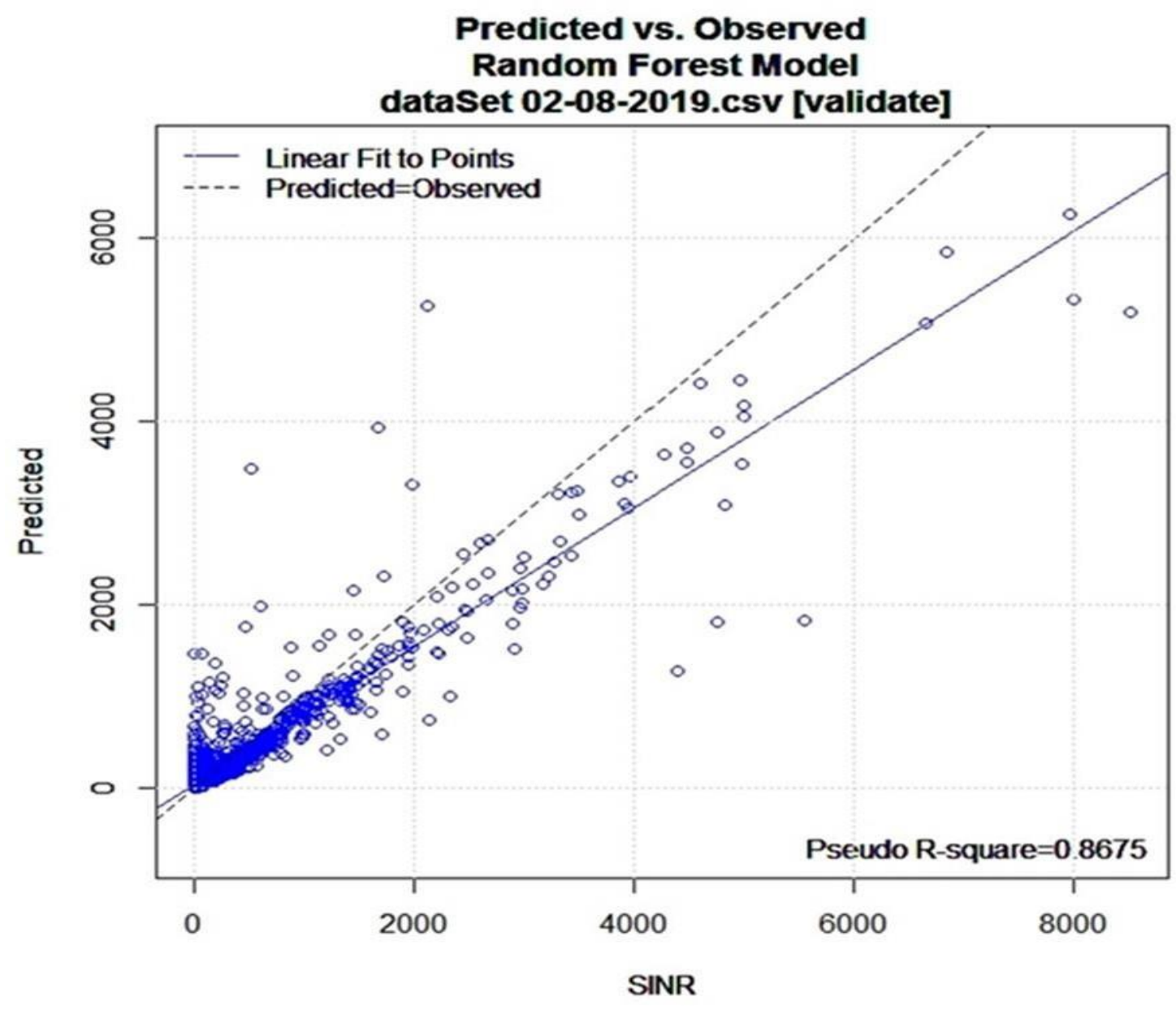

Fig. 7 Predicted vs. Observed in RF model during validation phase 


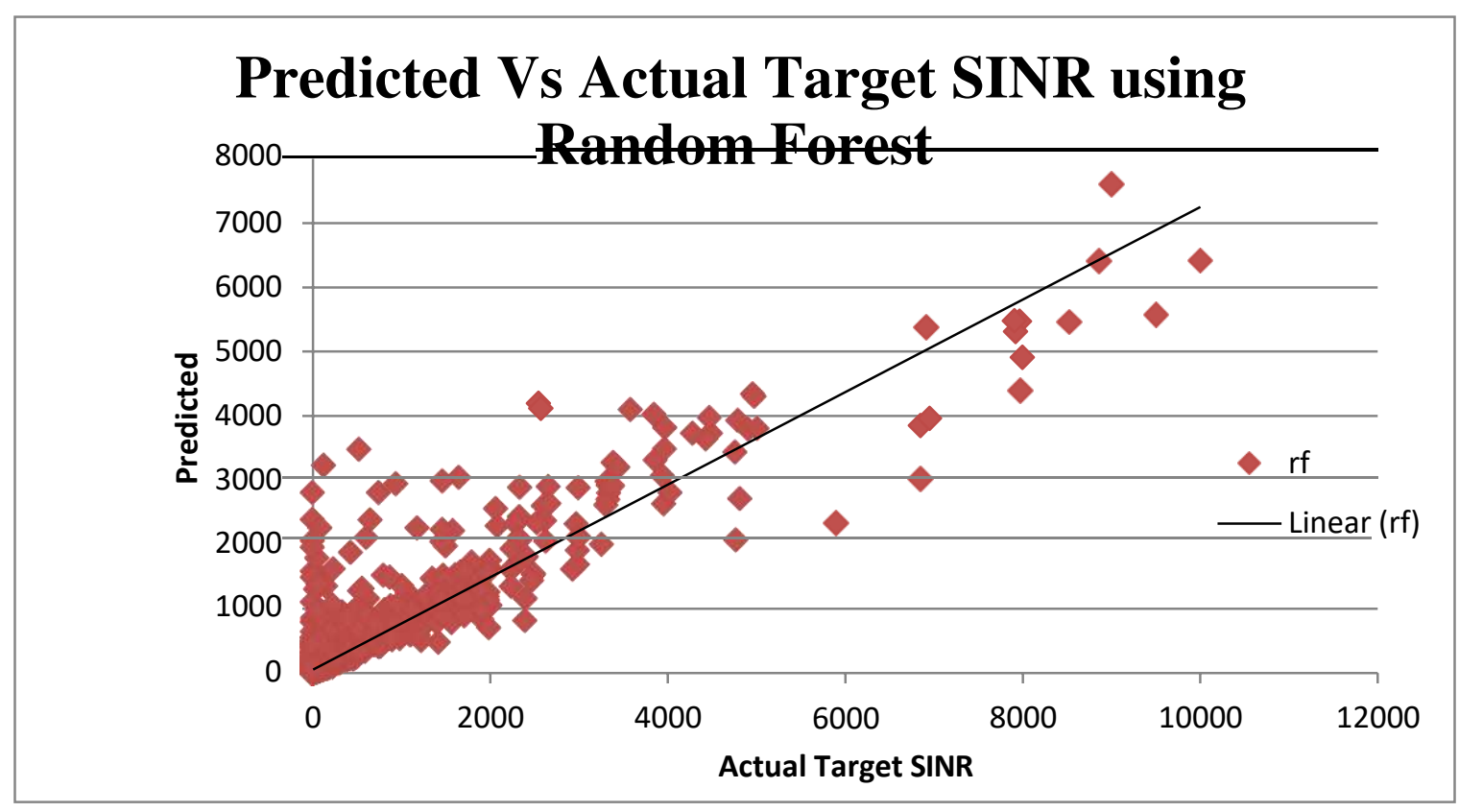

Fig. 8 Predicted vs. Observed in RF model during cross-validation phase

The observed SINR is plotted on the horizontal axis and predicted SINR on the vertical axis of the scatter plot. The location of each point on the graph depends on both the predicted and observed SINR values in figs. 5-8. The figs. 7 and 8 validate and crossvalidate the same conclusion-stronger; more linear and positive relationship between the predicted and observed SINR values using the RF model as compared to the other three ML models. The test set error is not utilized during the training phase. It is useful for comparing various models and may be plotted during the training process. If it reaches a minimum value than the validation set error for a particular iteration number, the data set is poorly divided in nature. It is very difficult to know the speed of the employed training algorithm, which depends upon various factors, such as the complexity of the problem, the number of data points used in the training set, the number of control weights and biases in the network, the target error, and whether the network is being utilized for pattern recognition or function approximation. 


\section{Conclusion}

$\mathrm{BF}$ is a noise mitigation scheme to improve the SINR ratio of received signals, and focus transmitted signals in desired spatial directions. The parameters of each path of multipath propagation model are cleaved into the corresponding channel gain and the DOA information in the channel matrix. Here, the adaptive BF is used under low and high SINR regime using ML in MSINR sense, and is more suited to massive MIMO systems than switched BF due to its capability to suppress interference and power consumption reduction. The ML models, namely Decision Tree, Random Forest, Linear Model and Neural Network are used to predict the target SINR responsible for BF. The optimization of antenna combining weights is based on MSINR value. The ML models are evaluated and compared in terms of performance analysis parameters, namely correlation, $\mathrm{R}^{2}$, Mean Absolute Error and \% Accuracy on a data set generated using the python package pyArgus. Random Forest ML model is the best among the four ML models used and has the best performance analysis features as follows: Correlation-0.92, $\mathrm{R}^{2}-0.85$, Mean Absolute Error-70.73 and \% Accuracy-86.40.. The further research is required to improve the coding to enhance the performance analysis results shown in this paper. The proposed adaptive BF system may be applied in VSCs, which is to be explored in the next research. The more advanced antenna arrays can be used to overcome the optimum halfwavelength limit of arbitrary configured planar antenna systems.

\section{Data Statement}

Data will be submitted after manuscript acceptance so that the proper citation may be included in the final publication. The generated dataset will be provided in a file named dataset.csv to the journal, as I am facing difficulty in uploading the same.

\section{Conflict of interests- No}

Declarations- All sections are relevant to the manuscript. 


\section{REFERENCES}

[1] https://github.com/zinka/arraytool and https://zinka.wordpress.com/, S.R. Zinka, Python package.

[2] Liu Z., J. He., "Linearly constrained minimum- 'normalised variance' beamforming against heavytailed impulsive noise of unknown statistics", IET Radar Sonar \& Navigation, vol. 2, issue 6, pp. 44957, 2008.

[3] Book chapter on "Recent advances in network beamforming" by Shahram ShahbazPanahi, Yindi Jing, in Academic Press Library in Signal Processing, Volume 7, 2018.

[4] Xiaotong Li, Ruiting Zhou, Ying-Jun Angela Zhang, Lei Jiao, Zongpeng Li, "Smart vehicular communication via 5G mmWaves”, Elsevier, Computer Networks, vol. 172, pp. 1-12, 2020.

[5] Dian Fan, Feifei Gao, Yuanwei Liu, Yansha Deng, Gongpu Wang, Zhangdui Zhong, Arumugam Nallanathan, “Angle Domain Channel Estimation in Hybrid mmWave Massive MIMO Systems”, IEEE Trans. on Wireless Communications, pp. 1-15, 2018.

[6] Mietzner J., Schober R., Lampe L., Gerstacker W.H., Hoeher P.A., "Multiple Antenna Techniques for Wireless Communication - a Comprehensive Literature Survey", IEEE Commun. Surv. and Tutorials, vol. 11, no. 2, pp. 87-105, 2009.

[7] Book chapter on "Performance of Multi-antenna Features and Configurations" by Henrik Asplund et al. Advanced Antenna Systems for 5G Network Deployments, 2020.

[8] Book chapter on" Cognitive Radio in Multiple-Antenna Systems” by Jae Moung Kim et al. in Cognitive Radio Technology (Second Edition), 2009.

[9] Dian Fan, Yansha Deng, Feifei Gao, Yuanwei Liu, Gongpu Wang, Zhangdui Zhong, Arumugam Nallanathan, "Training Based DOA Estimation in Hybrid mmWave Massive MIMO Systems", GLOBECOM 2017-2017 IEEE Global Commun. Conf., DOI: 10.1109/GLOCOM.2017.8254826, 2017.

[10] Fang X., W. Luo, M. Cheng, "Beamforming and Positioning-Assisted Handover Scheme for Long- Term Evolution System in High-Speed Railway", IET Communications, pp. 2335-40, 2012.

[11] David Munoz, Frantz Bouchereau, Cesar Vargas, Rogerio Enriquez, "Book on Position Location Techniques and Applications", published by Elsevier, 2009.

[12] Demosthenes Vouyioukas," A Survey on Beamforming Techniques for Wireless MIMO Relay Networks", Hindawi Publishing Corporation, International Journal of Antennas and Propagation, pp. 1-21, Volume 2013,

[13] Balanis C. A., “Antenna Theory Analysis and Design”, John Wiley \& Sons, New York, 1997. 
[14] Krous J. D.: Antenna, Mc GRAW-HILL, New York, 1950.

[15] Ehab Ali, Mahamod Ismail, Rosdiadee Nordin, Nor Fadzilah Abdulah, "Review: Beamforming Techniques for Massive MIMO Systems in 5G: Overview, Classification, and Trends for Future Research”, Zhejiang University and Springer-Verlag Berlin Heidelberg, pp. 753-72, 2017.

[16] Anita V., Sri Jaya Lakshmi S., Sreedevi I., Khan H., Sarat Kumar K., Ramakrishna P., “An Adaptive Processing of Linear Array for Target Detection Improve", Int. J. Comput. Appl. (09758887), vol. 42, no. 4, pp. 33-6, 2012.

[17] Mailloux R. J., "Phased Array Architecture for Millimetric Active Arrays", IEEE Antennas Propag. Soc. Newslett., vol. 28, pp. 4-7, 1986.

[18] Schrank H. E., "Low Sidelobe Phased Array Antennas", IEEE Antennas Propag. Soc. Newslett., vol. 25, no. 2, pp. 4-9, 1983.

[19] Applebaum S. P., Chapman D. J., "Adaptive Arrays with Main Beam Constraints", IEEE Trans. Antennas Propag., AI-24, pp. 650-62, 1976.

[20] Fangxiao Jin, Tianshuang Qiu, Tao Liu, "Robust Cyclic Beamforming against Cycle Frequency Error in Gaussian and Impulsive Noise Environments", Int. J. Electron. Commun. by Elsevier, pp. $153-60,2019$.

[21] Shepherd R. A., "Measurements of Amplitude Probability Distributions and Power of Automobile Ignition Noise at HF”, IEEE Trans. Veh. Technol., vol. 23, no. 3, pp. 72-83, 1974.

[22] Blackard K. L., Rappaport T. S., Bostian C. W., "Measurements and Models of Radio Frequency Impulsive Noise for Indoor Wireless Communications”, IEEE J. Sel. Areas Commun., pp. 991-1001, 1993.

[23] Tsakalides P., Nicias C. L., "Robust Space-time Adaptive Processing (STAP) in Non-Gaussian Clutter Environments”, IEE Proceedings-Radar, Sonar Navig., pp. 84-93, 1999.

[24] Kannan B., Fitzgerald W. J., "Beamforming in Additive A-Stable Noise using Fractional Lower Order Statistics (FLOS)”, Proc. IEEE Int. Conf. Electron. Circuits, Syst., pp. 1755-8, 1999.

[25] He J., Liu Z., Thomas Wong K., "Linearly Constrained Minimum- "Geometric Power" Adaptive Beamforming using Logarithmic Moments of Data containing Heavy-Tailed Noise of Unknown Statistics”, IEEE Antennas Wirel. Propag. Lett., vol. 6, pp. 600-3, 2007.

[26] Zhao H., Zhang Y., Li E-P, Buonanno A., D' Urso M., “Diagnosis of Array Failure in Impulsive Noise Environment using Unsupervised Support Vector Regression Method", IEEE Trans. Antennas Propag., vol. 61, no.11, pp. 5508-16, 2013. 
[27] Ma W., Qu H., Gui G., Xu L., Zhao J., Chen B., “Maximum Correntropy Criterion based Sparse

Adaptive Filtering Algorithms for Robust Channel Estimation under Non-Gaussian Environments", J. of the Franklin Institute, pp. 2708-28, 2015.

[28] Chen B., Liu X., Zhao H., Principe J. C., "Maximum Correntropy Kalman Filter Automatica”, pp. $70-7,2017$.

[29] Wu Z., Peng S., Chen B., Zhao H., "Robust Hammerstein Adaptive Filtering under Maximum Correntropy Criterion", Entropy, vol. 17, no. 12, pp. 7149-66, 2015.

[30] Hajiabadi M., Hodtani G.A., Khoshbin H., "Adaptive Multitask Network based on Maximum Correntropy Learning Algorithm", Int. J. Adapt Control Signal Process., vol. 31, no. 8, pp. 1232-41, 2017.

[31] Chen B., Xing L., Zhao H., Zheng N., Principe J.C., "Generalized Correntropy for Robust Adaptive Filtering", IEEE Trans. Signal

Process., vol. 64, no. 13, pp. 3376-87, 2016.

[32] Taras Maksymyuk, Juraj Gazda, Oleh Yaremko, Denys Nevinskiy, "Deep Learning Based Massive MIMO Beamforming for 5G Mobile Network", The 4th IEEE Int. Symposium on Wireless Systems, pp. 241-4, 2017.

[33] Vishnu V. Ratnam, Andreas F. Molisch, "Periodic Analog Channel Estimation Aided Beamforming for Massive MIMO Systems", IEEE Transactions on Wireless Communications, vol. 18, no. 3, pp. 1581- 94, 2019.

[34] Kuo-Chen Ho, Shang-Ho Tsai, "A Novel Multiuser Beamforming System with Reduced Complexity and Beam Optimizations", IEEE Transactions on Wireless Communications, vol. 18, no. 9, pp. 4544-57, 2019.

[35] Hang Li, Thomas Q. Wang, Xiaojing Huang, "Joint Adaptive AoA and Polarization Estimation using Hybrid Dual-Polarized Antenna Arrays”, IEEE Access, vol. 7, pp. 76353-66, 2019.

[36] Mohammed A. Almagboul, Feng Shu, Yaolu Qin, Xiaobo Zhou, Jin Wang, Yuwen Qian, Kingsley Jun Zou, Abdeldime Mohamed Salih Abdelgader, “An Efficient Hybrid Beamforming Design for Massive MIMO Receive Systems via SINR Maximization Based on an Improved Bat Algorithm", IEEE Access, vol. 7, pp.136545-58, 2019.

[37] Hedi Khammari, Irfan Ahmed, Ghulam Bhatti, Masoud Alajmi, "Spatio-Radio Resource Management and Hybrid Beamforming for Limited Feedback Massive MIMO Systems", J. of Electronics, DOI:10.3390/electronics8101061, pp. 1-25, 2019. 
[38] Ahmet M. Elbir, "CNN-Based Precoder and Combiner Design in mmWave MIMO Systems", IEEE Communications Letters, vol. 23, no. 7, pp. 1240-43, 2019.

[39] Lorenzo Combi, Umberto Spagnolini, "Adaptive Optical Processing for Wideband Hybrid Beamforming”, IEEE Transactions on Communications, vol. 67, no. 7, pp. 4967-79. 2019.

[40] Susnata Mondal, Jeyanandh Paramesh, "A Reconfigurable 28-/37-GHz MMSE-Adaptive HybridBeamforming Receiver for Carrier Aggregation and Multi-Standard MIMO Communication", IEEE Journal of Solid-State Circuits, vol. 54, no. 5, pp. 1391-406, 2019.

[41] Linlin Sun, Yaolu Qin, Zhihong Zhuang, Riqing Chen, Yijin Zhang, Jinhui Lu, Feng Shu, Jiangzhou Wang, "A Robust Secure Hybrid Analog and Digital Receive Beamforming Scheme for Efficient Interference Reduction”, IEEE Access, vol. 7, pp. 22227-34, 2019.

[42] Li Zhu, Shilian Wang, Jiang Zhu, "Adaptive Beamforming Design for Millimeter-Wave Line-ofSight MIMO Channel”, IEEE Communications Letters, vol. 23, no. 11, pp. 2095-98, 2019.

[43] Yanan Liu, Xianbin Wang, Gary Boudreau, Akram Bin Sediq, Hatem Abou-zeid, "Deep Learning Based Hotspot Prediction and Beam Management for Adaptive Virtual Small Cell in 5G Networks", IEEE Transactions on Emerging Topics in Computational Intelligence, vol. 4, no. 1, pp. 83-94, 2020.

[44] Song Noh, Michael D. Zoltowski, David J. Love, "Multi-Resolution Codebook and Adaptive Beamforming Sequence Design for Millimeter Wave Beam Alignment", IEEE Transactions on Wireless Communications, vol. 16, no. 9, pp. 5689-501, 2017.

[45] Shi Q., Razaviyayn M., Luo Z.Q., and He C., "An Iteratively Weighted MMSE Approach to Distributed Sum-Utility Maximization for a MIMO Interfering Broadcast Channel," IEEE Trans. Signal Process., vol. 59, no. 9, pp. 4331-40, 2011.

[46] Yaohua Sun, Mugen Peng, Yangcheng Zhou, Yuzhe Huang, Shiwen Mao, "Application of Machine Learning in Wireless Networks:

Key Techniques and Open Issues”, IEEE Commun. Surv. and Tutorials, pp.1-37, 2019.

[47] Mingyi Hong, Zhi-Quan Luo, "Joint Linear Precoder Optimization and Base Station Selection for an Uplink MIMO Network: a Game Theoretic Approach", 2012 IEEE Int. Conf. on Acoustics, Speech and Signal Processing (ICASSP), pp. 2941-44, 2012.

[48] Bjornson E., Bengtsson M., Ottersten B., "Optimal Multiuser Transmit Beamforming: A Difficult Problem with A Simple Solution Structure," IEEE Signal Processing Magazine, vol. 31, no. 4, pp. 142 8, 2014. 
[49] Balanis C. A., “Antenna Theory: Analysis and Design”, 3rd ed.; Wiley-Interscience: New York, NY, USA, 2005.

[50] Monzingo R. A., Miller, T. W., "Introduction to Adaptive Arrays", Scitech. Publishing Inc.: Raleigh, NC, USA, 2004.

[51] Haykin S., “Adaptive Filter Theory”, 3rd ed.; Prentice Hall: Upper Saddle River, NJ, USA, 1996.

[52] Abhinav Sharma, Sanjay Mathur, "Performance Analysis of Adaptive Array Signal Processing Algorithms", IETE Technical Review, pp. 1-20, DOI: 10.1080/02564602.2015.1088411, 2016.

[53] Quinlan J. R., "Induction of Decision Trees", Machine learning, vol. 1, no. 1, pp. 81-106, 1986.

[54] Liaw A, Wiener M., "Classification and Regression by Random Forest", R News, vol. 2/3: pp.18$22,2002$.

[55] Chambers J. M., "Computational Methods for Data Analysis", Applied Stat., vol. 1, no. 2, pp.1$10,1977$.

[56] Riedmiller M., Braun H., "A Direct Adaptive Method for Faster Backpropagation Learning: The RPROP algorithm”, IEEE Int. Conf. on Neural Nets, pp. 586-91, 1993.

[57] Prashant Singh Rana, Harish Sharma, Mahua Bhattacharya, Anupam Shukla. "Quality assessment of modeled protein structure using physicochemical properties", Journal of Bioinformatics and Computational Biology, vol. 13, no. 2, pp. 1-19, 2015.

[58] Francisco Hugo Costa Neto Daniel Costa Araújo Tarcisio Ferreira Maciel, "Hybrid beamforming design based on unsupervised machine learning for millimeter wave systems", John Wiley \& Sons, Ltd., pp. 1-18, 2020.

[59] Hyung Jun Kwon, Jung Hoon Lee, and Wan Choi, "Machine Learning-Based Beamforming in Two- User MISO Interference Channels", ICAIIC, IEEE, pp. 496-99, 2019.

\section{Author}

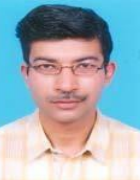

Sanjeev Chopra received his B.E. degree in Electronics and Instrumentation Engineering from Punjabi University, Punjab, India in 1997; M. Tech. degree in Electronics and Communication Engineering from Punjab Technical University, Punjab, India in 2010. He has twenty years' teaching experience from July 1997 to July 2017. He has published 12 research papers in various reputed Journals and Conferences. He is presently a Ph.D. scholar in Electronics and Communication Engineering

Department of Thapar Institute 
of Engineering and Technology, Patiala, Punjab, India. His research areas are Wireless Communication, Image Processing and Biomedical Electronics. 
Figures

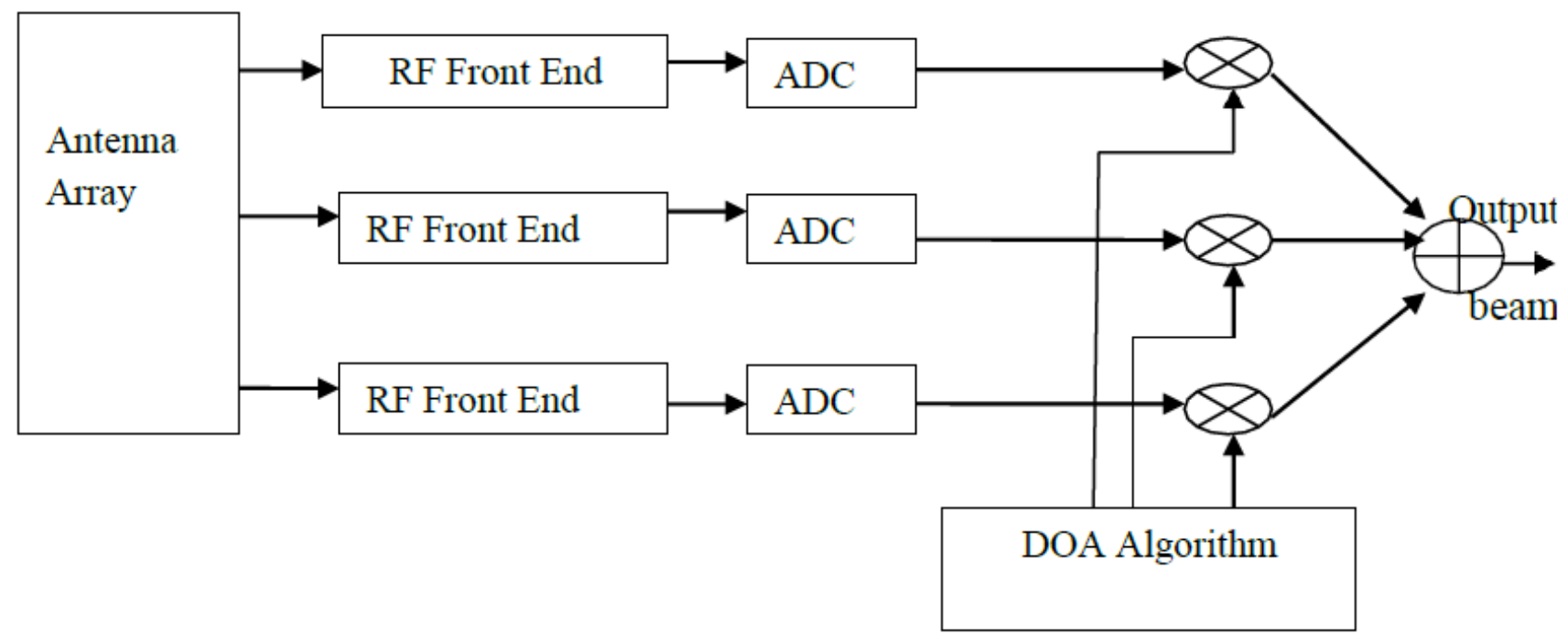

Figure 1

General Smart Antenna Array System

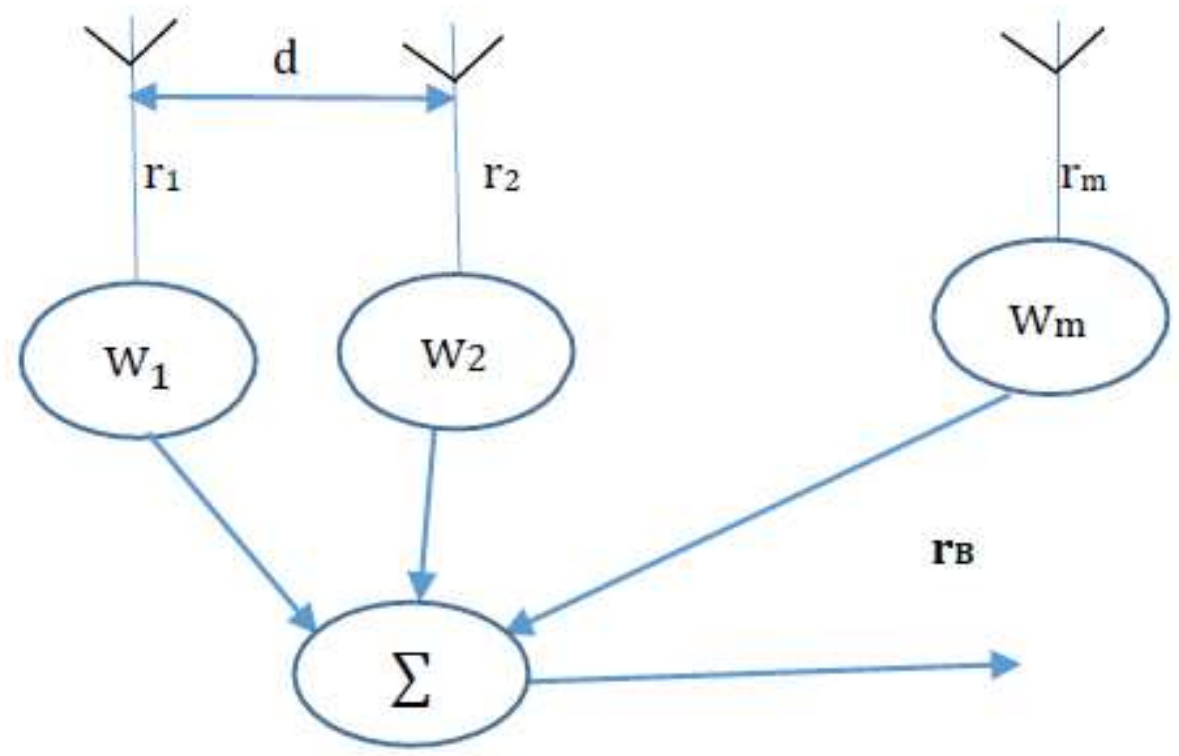

Figure 2

Working Principle of Beamformer 


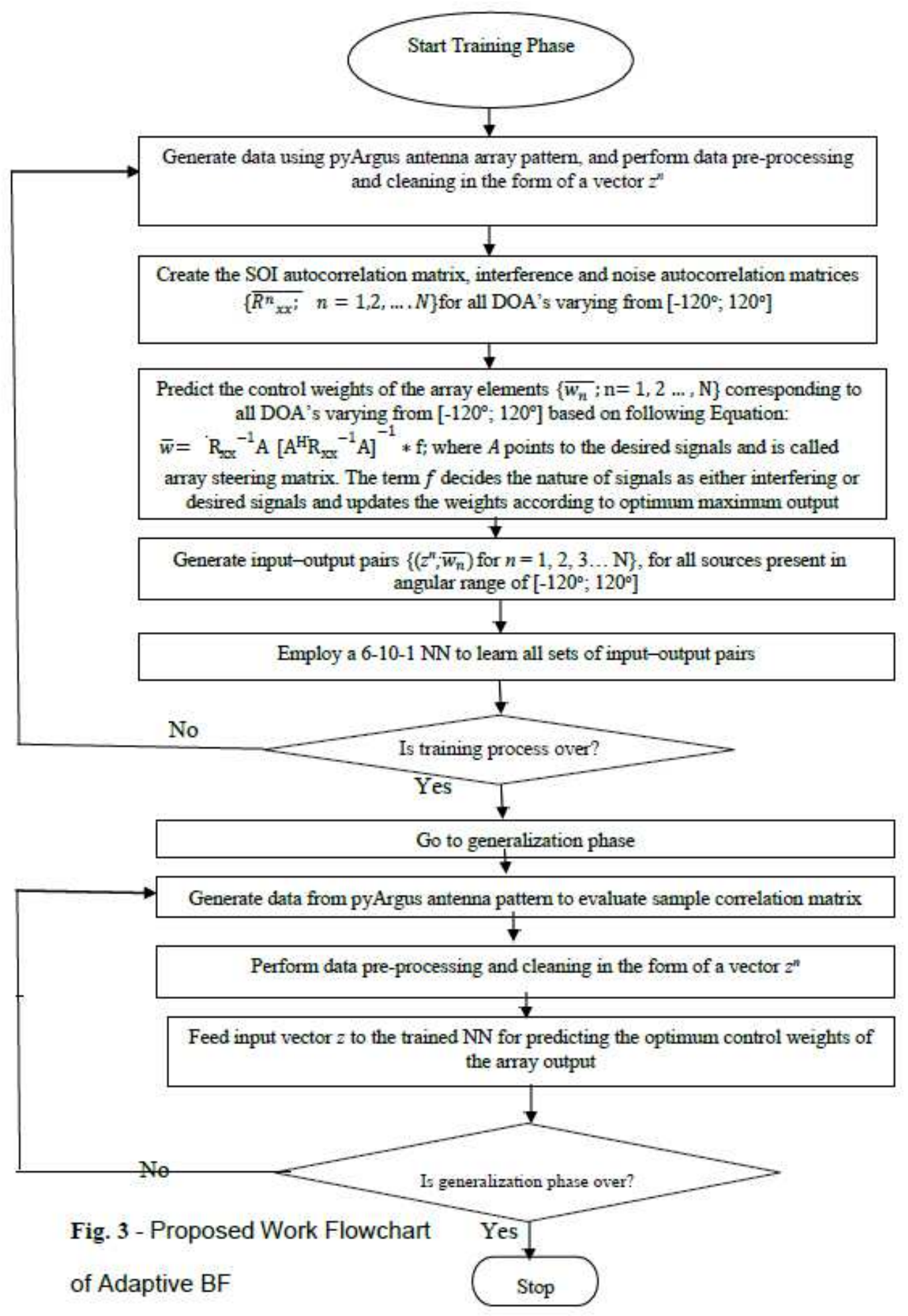

\section{Figure 3}




\begin{tabular}{|c|c|c|c|}
\hline $\begin{array}{l}\text { Data collection through } \\
\text { Modeling and } \\
\text { Simulation of python- } \\
\text { based signal processing } \\
\text { algorithms applicable in } \\
\text { antenna arrays } \\
\text { (pyArgus) }\end{array}$ & $\begin{array}{l}\text { Perform data } \\
\text { pre-processing } \\
\text { and cleaning }\end{array}$ & $\begin{array}{c}\text { Calculate } \\
\text { features of } \\
\text { importance } \\
\text { namely, } P_{\text {noise }}, d, \\
N, \text { theta_soi, } a \\
\text { and } b \text {. }\end{array}$ & $\begin{array}{l}\text { Model building } \\
\text { on training- } \\
\text { testing dataset }\end{array}$ \\
\hline & & $\begin{array}{l}\text { Result analysis } \\
\text { having } \\
\text { Validation and } \\
\text { Discussion }\end{array}$ & $\begin{array}{l}\text { Calculate evaluation } \\
\text { parameters namely } \\
\text { MAE, } R, R^{2} \text { and } \% \\
\text { Accuracy on training- } \\
\text { testing dataset }\end{array}$ \\
\hline
\end{tabular}

Figure 4

Various Steps of Methodology used 

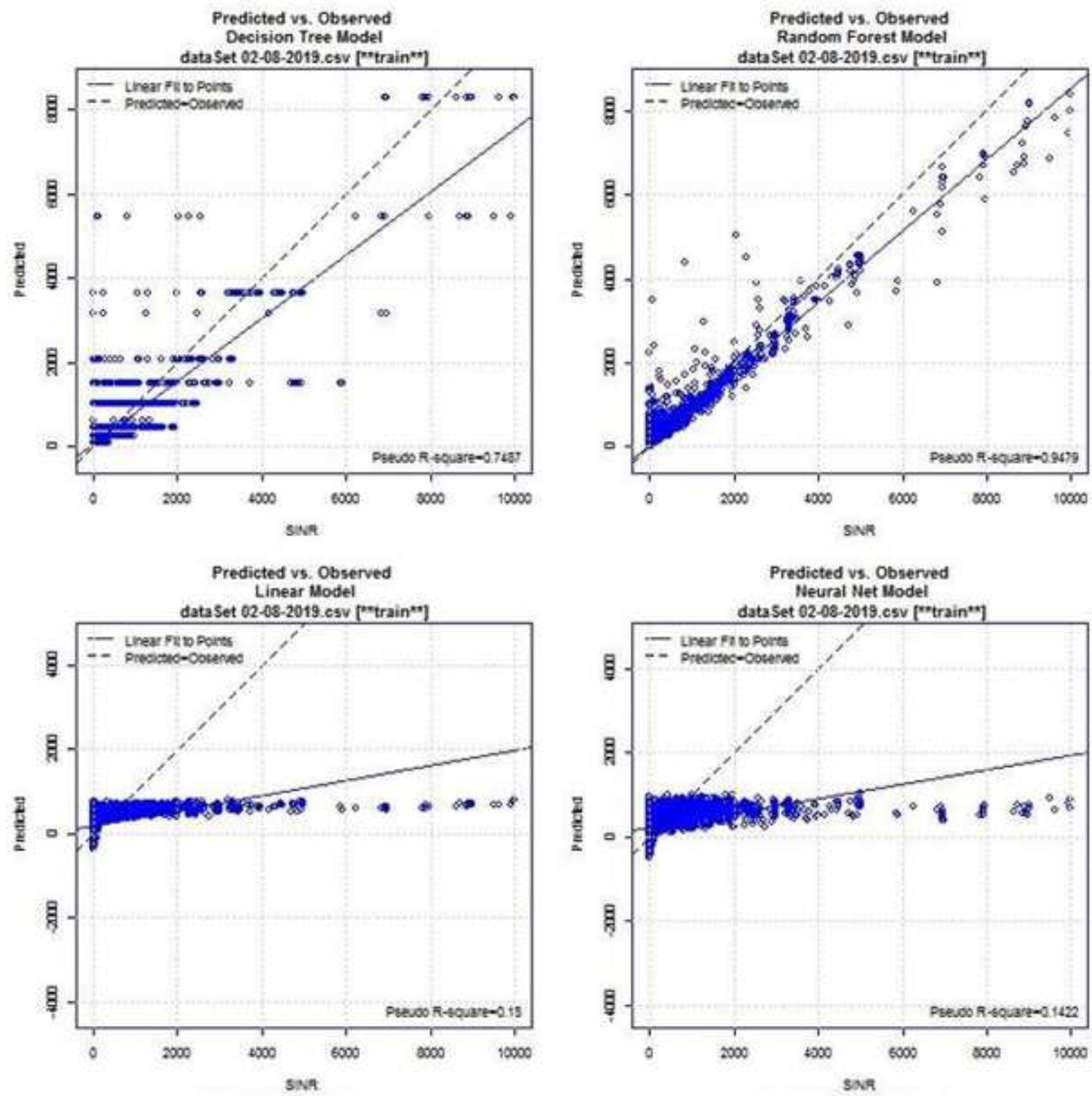

Figure 5

(a) Predicted vs. Observed Decision Tree Model (b) Predicted vs. Observed Random Forest Model (c) Predicted vs. Observed Linear Model (d) Predicted vs. Observed Neural Net Model on Training dataset 

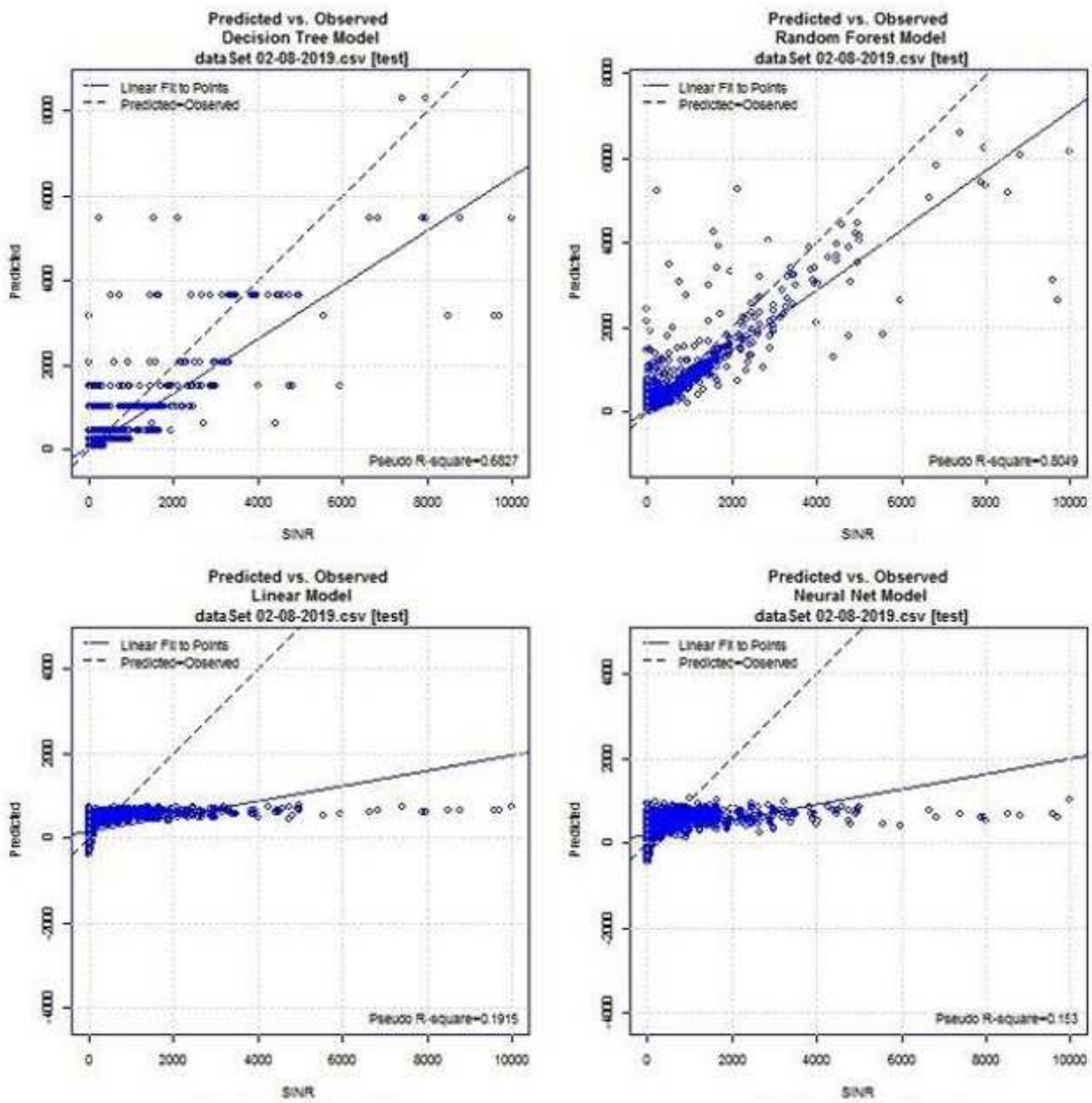

Figure 6

(a) Predicted vs. Observed Decision Tree Model (b) Predicted vs. Observed Random Forest Model (c) Predicted vs. Observed Linear Model (d) Predicted vs. Observed Neural Net Model on Testing dataset 


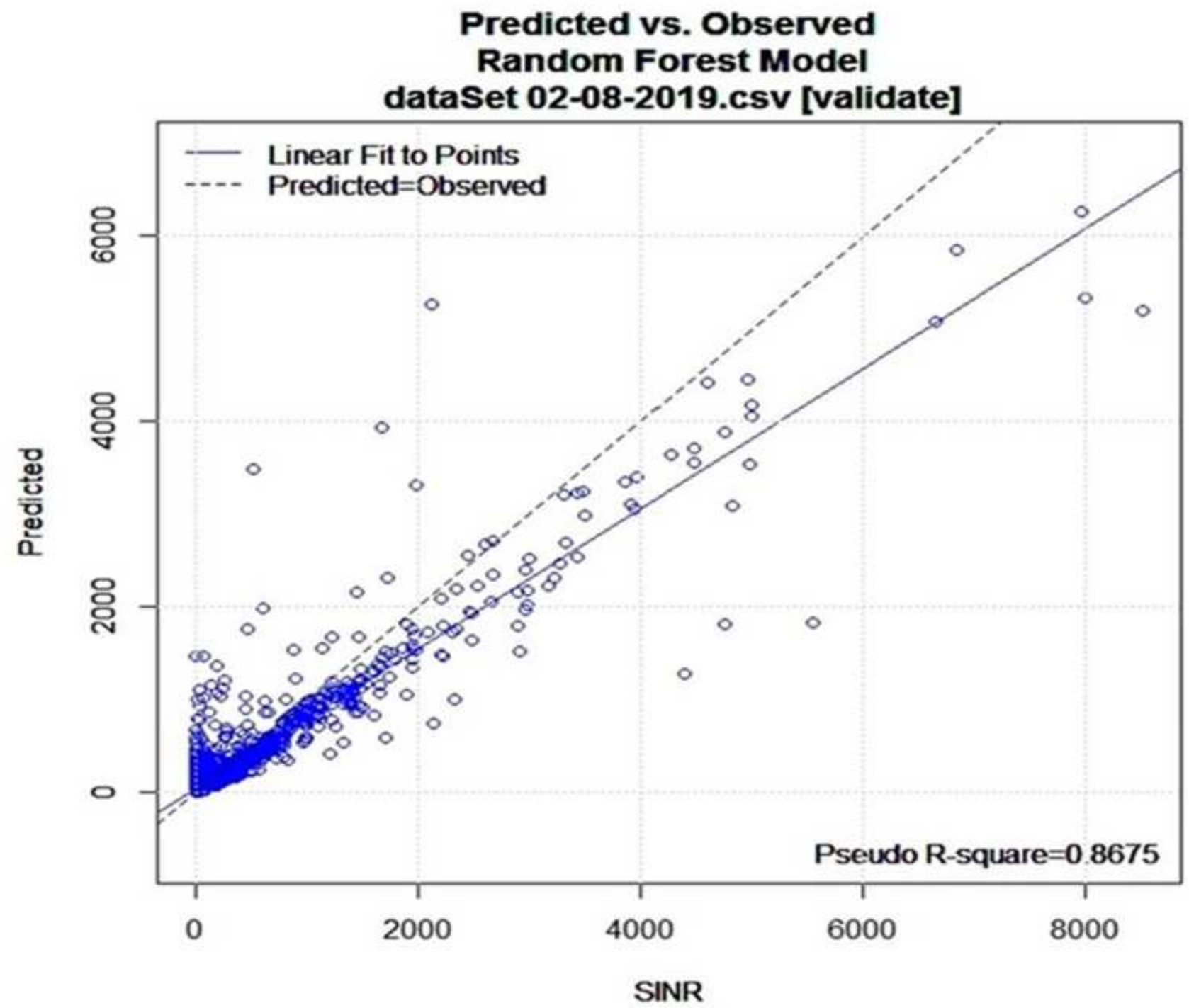

Figure 7

Predicted vs. Observed in RF model during validation phase 


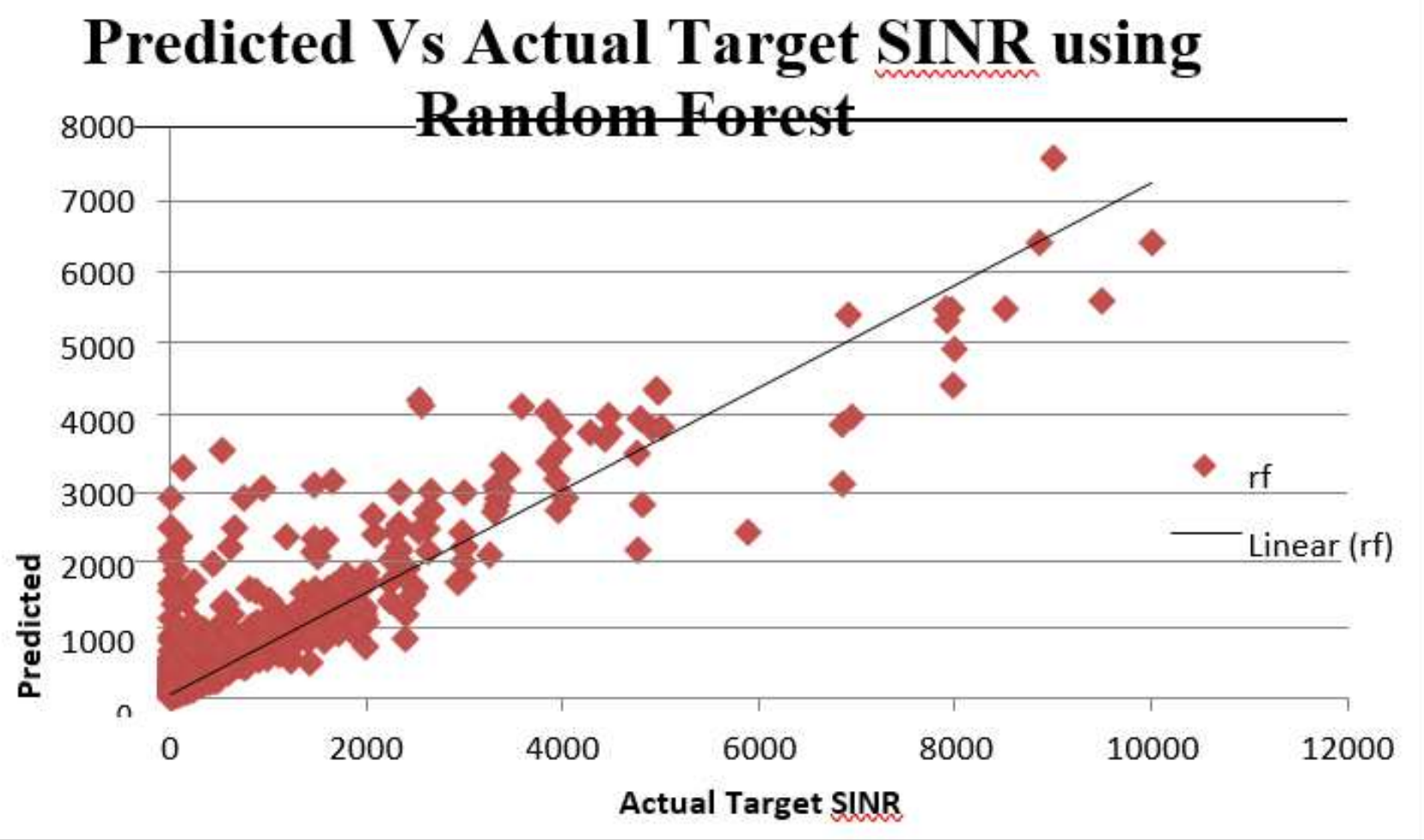

Figure 8

Predicted vs. Observed in RF model during cross-validation phase 